\title{
Desingularization of Implicit Analytic Differential Equations
}

\author{
Hernán Cendra $^{a}$, María Etchechoury $^{b, 1}$ and Alberto Ibort ${ }^{c}$ \\ a Universidad Nacional del Sur, Av. Alem 1253 \\ 8000 Bahía Blanca and CONICET, Argentina. \\ uscendra@criba.edu.ar \\ b Laboratorio de Electrónica Industrial, Control e Instrumentación, \\ Facultad de Ingeniería, Universidad Nacional de La Plata and \\ Departamento de Matemática, Facultad de Ciencias Exactas, \\ Universidad Nacional de La Plata. \\ CC 172, 1900 La Plata, Argentina. \\ marila@mate.unlp.edu.ar \\ c Departamento de Matemáticas, Universidad Carlos III de Madrid, \\ Av. de la Universidad 30, Leganés, Madrid, Spain. \\ albertoi@math.uc3m.es
}

\begin{abstract}
The question of finding solutions to given implicit differential equations (IDE) has been answered by several authors in the last few years, using different approaches, in an algebraic and also a geometric setting. Many of those results assume in one way or another that the subimmersion theorem can be applied at several stages of the reduction algorithm, which, roughly speaking, allows to reduce a given IDE to a collection of ODE depending on parameters. The main purpose of the present paper is to improve some of the known results by introducing at each stage of the reduction algorithm a desingularization of the manifolds with singularities that may appear when the subimmersion theorem cannot be applied. This can be done for analytic IDE by using some fundamental results on subanalytic subsets and
\end{abstract}

${ }^{1}$ Corresponding author. Fax: $+54-221-424-5875$ 
desingularization of closed subanalytic subsets due mainly to Lojasiewicz, Hironaka, Gabrielov, Hardt, Bierstone, Milman and Sussmann, among others. We will show how this approach helps to understand the dynamics given by the LagrangeD'Alembert-Poincaré equations for the symmetric elastic sphere.

Keywords: Implicit differential equations; Differential-algebraic systems; Desingularization; Nonholonomic systems.

\section{Introduction}

Implicit differential equations $\phi(x, \dot{x})=0$ (IDE) are very common in science and technology. The case of an ODE $\dot{x}=f(x)$ is the simplest particular case. The Euler-Lagrange equations for a given Lagrangian, degenerate or not [1, 2, 3]; Lagrange-D'Alembert equations for a nonholonomic system and their reduced versions Lagrange-D'AlembertPoincaré equations [4, 5, 6]; some equations in the Dirac theory of constraints [7]; electrical circuits called descriptor or semistate systems [8] electric power systems [9]; nonstandard singularly perturbed systems [10; reactive columns [11; constrained robot systems [12, are some of the examples.

In the literature, IDE are often called differential algebraic systems (DAS) in the case in which the subimmersion theorem can be applied, in one way or another, at each stage of the so called reduction algorithm, 13. In the last few years this approach to finding the solutions to a given DAS in the category of $C^{\infty}$ manifolds and maps, and even some more general IDE, has been intensively studied. Invariants like the so called index of the system were introduced and calculated, as far as it is possible, for a general DAS and geometrically motivated algorithms have been developed, see, for instance, [13, 14, 15, 16, 17, 18, just to mention part of the relevant bibliography more or less connected to the present work. This kind of approach has now reached a nearly optimal answer to certain basic questions, for instance, the question about existence and uniqueness of solutions for DAS.

One may say that the reduction algorithm, as exposed in the references cited above, uses some of the old ideas of the Dirac theory of constraints [7, but generalizes them to make them useful in a variety of fields beyond Hamiltonian and quantum mechanics, like control theory and nonholonomic mechanics, as it appears, for instance, in the references cited above.

To the best of our knowledge, the singular cases where the subimmersion theorem can- 
not be applied have not been systematically and fully studied in the existing literature, although there are several related papers. For instance, an algebraic approach relying on complex algebraic geometry is presented in [19]. In that article the singular cases where the subimmersion theorem cannot be applied are treated in the case of implicit differential equations given by complex polynomial relations. Also an implementation using computer algebra systems is provided. Besides [19], cited above, there are several papers studying specific questions about singular points and impasse points, see, for instance, 20, 21, 22, 23. See also [4] for examples in nonholonomic mechanics where a simple example of desingularization appears.

Desingularization ideas appear in different contexts in mathematics. The fundamental theorem of Hironaka 24], whose proof was simplified and computationally implemented in subsequent works [25, 26], is a good example of a desingularization procedure, in this case desingularization of certain algebraic varieties. Another fundamental theorem on desingularization was proven by Bierstone and Milman [27, which actually includes Hironaka's theorem. See also [28].

In the present work we are going to use some of the known desingularization results, and also several results from the theory of subanalytic sets. Our main result shows how to reduce a given real analytic IDE to a real analytic IDE of constant rank, defined in section 2, which is considered the simplest case in this paper. We do this by conveniently modifying the usual reduction algorithm by including a desingularization of all the singular manifolds that may appear at each stage of the algorithm. The only main result on desingularization that we use for doing this is the theorem of Hironaka on desingularization of closed subanalytic sets [29]. In fact, the theorem on desingularization of closed analytic subsets, theorem 5.1 of Bierstone and Milman in [30, is enough for our purposes. We will keep the conceptual framework as basic as possible throughout the paper, avoiding the unnecessary usage of schemes.

Control systems in the category of subanalytic sets have been recently studied in 31, where desingularization techniques, exposed, for instance, in [30] and 32, have been used. In the present work we also work, in a sense, with control systems in the category of subanalytic sets, but from the point of view of IDE, which in a sense is dual to the point of view of control theory. In fact, a control system is, roughly, a vector field depending on a parameter, or equivalently, a family of vector fields, or, more generally, a family of local vector fields. On the other hand, as we will see, an IDE of constant rank gives also a family of vector fields, but defined implicitly.

Working with IDE in the subanalytic category rather than in some of the $C^{k}, k=1,2 \ldots \infty$ 
categories, of course is a limitation, but there are interesting examples that can be studied in this context. In fact, in the study of IDE representing several important examples from mechanics, control theory and other fields, as we have mentioned before, equations are often given by real analytic functions.

The relevance of the desingularization method that we propose to help finding the solutions to a given IDE depends, in part, on a sufficient knowledge of the geometry of the resulting desingularizing manifold. For instance, if in a given example the final system obtained by the methods of the present paper is simply a vector field, existence of equilibrium points may be directly related to the topology of the desingularizing manifold.

We develop an interesting example showing how the desingularization method helps to solve a mechanical system, namely, the rolling symmetric elastic sphere. By definition, this system has an extra nonholonomic condition besides the usual nonsliding condition for a rigid rolling sphere with only one point of contact with the plane. This extra condition says that the vertical component of the angular velocity should be 0 . A physical situation corresponding approximately to this model is that of an elastic sphere, like, for instance, a rubber sphere, which is slightly deformed as it rolls on the floor. We assume that the deformation of the sphere takes place only on that part of the sphere in contact with the floor. Then there is an area of contact, rather than a point of contact, which imposes, because of the nonsliding condition, the condition that the vertical component of the angular velocity is 0 . From the point of view of reduction theory, this condition introduces singularities in the reduced system of equations, since the standard dimension assumption for the Lagrange-D'Alembert-Poincaré equations is only partially satisfied, see [5]. The nonholonomic condition that the vertical component of the angular velocity is 0 has been considered for the example of a rigid body with a fixed point in 33. We must say that the model that we are considering for a rolling symmetric elastic sphere is not necessarily very realistic because it does not takes into account, for instance, the deformation that may occur in the material far from the area of contact, or the viscoelasticity, which introduces internal friction. Those are delicate problems in elasticity theory which have been the subject of several investigations for more than a century and are an active area of research in our days, but this is not the subject of our paper. We will concentrate only on the dynamical equations for a rolling sphere under the specified nonholonomic conditions. This kind of system has potential applications to robotics. The desingularization process can be performed in detail in the case of the symmetric elastic sphere and the desingularizing manifolds can be identified, which leads directly to solving the system by quadratures. In fact, we show that the original system is equivalent to a system on the nonsingular manifold $S^{2} \times S^{1}$. In spite of the vast literature on the subject of nonholonomic systems with rolling constraints we believe that our results 
in the specific case of the symmetric elastic sphere are new and interesting by themselves.

We will work mainly in the category of subanalytic sets and maps. Manifolds will be usually real analytic manifolds and maps from one manifold to another will be real analytic maps, although some of the statement are also valid in a more general context.

In section 2 we explain some basic facts about IDE. In section 3 we describe our algorithm. In section 4 we prove our main results. In section 5 we give a detailed description of the example of the symmetric elastic sphere.

\section{Implicit differential equations}

Basic notation. In this section manifolds will be smooth manifolds and maps from one manifold to another will be smooth maps. Let $M$ be a given manifold of dimension $n$ and $F$ a vector space of dimension $m$. Let $a: T M \rightarrow F$ be a map such that, for each $(x, \dot{x}) \in T M, a(x, \dot{x}) \equiv a(x) \dot{x} \equiv a(x)(x, \dot{x})$ is linear in $\dot{x}$. Let $f: M \rightarrow F$ be a given map. We will study IDE of the type

$$
a(x) \dot{x}=f(x)
$$

By introducing the trivial vector bundle $M \times F$ we can think of $a$ as representing a vector bundle map

$$
a: T M \rightarrow M \times F
$$

and of $f$ as being a section of $M \times F$. Then, for each $x \in M, a(x)$ is a linear map depending smoothly on $x$ from the tangent space $T_{x} M$ into the fiber $(x, F)$ of the trivial bundle. More generally, we may consider a general vector bundle with base $M$, say $\pi: F \rightarrow M$ and an IDE like (2.1) where now $a: T M \rightarrow F$ is a vector bundle map and $f$ is a section of $F$. This kind of generalization is important to describe a sufficiently wide class of IDE. However, in the present paper we shall describe only the trivial bundle case, for simplicity and also because it already contains the essential facts. The general case can be treated in an essentially similar way. In this paper the manifold $M$ is called the domain and the space, or more generally, vector bundle $F$, is called the range of the IDE.

Given an IDE one has immediately a linear algebraic system (LAS) for each $x \in M$, depending smoothly on $x$, where the unknown is $(x, \dot{x})$, for each $x \in M$. We will call it the $L A S$ associated to the given IDE. 
IDE of constant rank. Assume that the LAS associated to (2.1) has solution $(x, \dot{x})$ for each $x \in M$. Then (2.1) defines an affine distribution, generally singular, on $M$. If, in addition, $\operatorname{rank} a(x)=\operatorname{rank}[a(x), f(x)]$ is locally constant, that is, it is constant on each connected component of $M$, then the IDE is called an IDE of constant rank. This is equivalent to saying that the corresponding affine distribution has constant rank on each connected component of $M$. For instance, if $m=n$ and $a(x)$ is invertible for all $x \in M$ then (2.1) is equivalent to an ODE

$$
\dot{x}=a(x)^{-1} f(x)
$$

and the rank of the affine distribution is 0 in this case.

The case of an IDE of constant rank is the simplest case in our context and our main result, in section 4, shows that a given analytic IDE can be reduced to a finite collection of analytic IDE of constant rank, which can be considered also as a single IDE of constant rank in an obvious way.

Reduction of a general IDE to an IDE of the type (2.1). It is easy to see that, from the point of view of a general theory where the dimension does not plays an essential role, IDE of the type

$$
\phi(x, \dot{x})=0
$$

where the map $\phi: T M \rightarrow F$ may be nonlinear in $\dot{x}$ are not more general than (2.1).

In fact, let us assume first, for simplicity, that $M$ is an open subset of a finite dimensional vector space $E$. An IDE of the type

$$
\phi(x, \dot{x})=0
$$

can be rewritten in the form (2.1) with domain $M \times E$ and range $E \times F$ as follows

$$
\begin{aligned}
\dot{x} & =u \\
0 & =\phi(x, u),
\end{aligned}
$$

which has the form (2.1) with

$$
a(x, u)=\left[\begin{array}{ll}
I & 0 \\
0 & 0
\end{array}\right], f(x, u)=\left[\begin{array}{c}
u \\
\phi(x, u)
\end{array}\right] .
$$

The case of a general manifold $M$ and a fiber preserving map $\phi: T M \rightarrow F$ where $F$ is a vector bundle with base $M$ can be also reduced to the form (2.1) by an essentially 
similar procedure, using a geometric construction involving pull-backs of bundles.

Remark. (i) We must remark that working with IDE written in the form (2.1) is an important ingredient of our algorithm, in part because this form is preserved and the space $F$ remains the same (or the vector bundle is replaced by a pull-back vector bundle) at each stage of the algorithm, which simplifies matters as will become evident later.

(ii) The system (2.1) can be written equivalently as follows

$$
\begin{array}{r}
a(x) \dot{x}=\dot{t} f(x) \\
\dot{t}=1,
\end{array}
$$

where $t=t(s)$. The first equation of this system can be written equivalently as follows

$$
b(y) \dot{y}=0
$$

where $y=(x, t), b(y) \in L(T(M \times \mathbb{R}), F), b(y)=[a(x),-f(x)]$.

Of course (2.3) is an IDE whose associated LAS has a solution for each $y$, but it does not seem that questions like reachability for (2.1) could be easily reduced to easily solvable corresponding questions for (2.3). In other words, this kind of transformation of the system does not necessarily really simplifies the problems related to a given IDE. On the other hand, systems like (2.3) are interesting by themselves and are related to Pfaffian systems 34].

Some notation and operations with IDE. It will be convenient to denote $(a, f)$ the IDE (2.1), from now on. Let $(a, f)$ be a given IDE, say

$$
a: T M \rightarrow F, \quad f: M \rightarrow F
$$

Let $N \subseteq M$ be a given submanifold. The restriction $(a, f) \mid N$, also written $(a|N, f| N)$, of $(a, f)$ to $N$ is defined naturally by the conditions $(a \mid N)(x)(x, \dot{x})=a(x)(x, \dot{x})$ and $(f \mid N)(x)=f(x)$, for all $(x, \dot{x}) \in T N$.

Let us remark that the notion of restriction $(a, f) \mid N$ makes sense also in the case where $N$ is any subset of $M$. In fact, we only need to give a meaning to the notion of a tangent vector $\left(x_{0}, \dot{x}\right)$ at a point $x_{0} \in N$. It is simply the tangent vector in $T_{x_{0}} M$ to a smooth curve $x(t), t \in(-\delta, \delta)$ in $M$ such that $x(t) \in N$, for all $t \in(-\delta, \delta)$ and $x(0)=x_{0}$.

Let $\varphi: N \rightarrow M$ be a given map. Then the pull-back $\varphi^{*}(a, f)=\left(\varphi^{*} a, \varphi^{*} f\right)$ is the IDE with domain $N$ and range $F$ defined by $\varphi^{*} a(y)(y, \dot{y})=a(\varphi(y))(T \varphi(y, \dot{y}))$ and 
$\varphi^{*} f(y)=f(\varphi(y))$

If $g: F \rightarrow G$ is a linear map we define the projection of $(a, f)$ by $g$ as being the IDE with domain $M$ and range $G$ defined by $(g \circ a, g \circ f)$. More precisely, we have $(g \circ a)(x)(x, \dot{x})=g(a(x)(x, \dot{x}))$ and $(g \circ f)(x)=g(f(x))$, for all $(x, \dot{x}) \in T M$.

One can define operations like the direct sum $\oplus$ or tensor product $\otimes$ of IDE in a natural way. For instance, if $\left(a_{i}, f_{i}\right), i=1,2$ are given IDE with domain $M$ and range $F_{i}$, $i=1,2$, then we can define the direct sum $\left(a_{1}, f_{1}\right) \oplus\left(a_{2}, f_{2}\right) \equiv\left(a_{1} \oplus a_{2}, f_{1} \oplus f_{2}\right)$ as an IDE with domain $M$ and range $F_{1} \oplus F_{2}$ by $\left(a_{1} \oplus a_{2}\right)(x) \dot{x}=a_{1}(x) \dot{x} \oplus a_{2}(x) \dot{x}$, and $\left(f_{1} \oplus f_{2}\right)(x)=f_{1}(x) \oplus f_{2}(x)$, for all $(x, \dot{x}) \in T M$. The tensor product is also defined in a natural way.

Using operations like the ones described above, one may sometimes simplify a given IDE. For instance, working in coordinates in $F$, say $\left(y_{1}, y_{2}, \ldots, y_{m}\right)$, if some of the equations, say corresponding to $y_{1}$, is a linear consequence of the others then it can be eliminated by using a projection $g\left(y_{1}, y_{2}, \ldots, y_{m}\right)=\left(y_{2}, \ldots, y_{m}\right)$, and the resulting system will be equivalent to the given one. We will need the following result, whose proof is not difficult.

Theorem 2.1 Let $(a, f)$ be a given IDE with domain $M$ and range $F$ and let $N \subseteq M$ be a given submanifold defined regularly by equations $\varphi=0$, where $\varphi: M \rightarrow H$ and $H$ is a finite dimensional vector space. Then the restriction $(a, f) \mid N$ has the same solutions as the IDE $(a \oplus 0, f \oplus \varphi)$ with domain $M$ and range $F \oplus H$. It also has the same solutions as the IDE $(a \oplus D \varphi \oplus 0, f \oplus 0 \oplus \varphi)$ with domain $M$ and range $F \oplus H \oplus H$. Here $D \varphi: T M \rightarrow H$ is defined by $D \varphi=p_{2} \circ T \varphi$, where $p_{2}: H \times H \rightarrow H$ is the projection on the second factor and $T H \equiv H \times H$.

This theorem is simple but useful. For instance, it allows sometimes to replace a given IDE by an equivalent IDE whose domain and range are vector spaces, which sometimes simplifies practical calculations avoiding the usage of local charts whenever it is convenient. More precisely, let a given $\operatorname{IDE}(a, f)$ having domain $M \subseteq L$, imbedded in the vector space $L$ and defined regularly by an equation $\varphi=0$, where $\varphi: L \rightarrow H$, and let $a$ be defined by a restriction $a=A \mid T M$, where $A: T L \rightarrow F$. Then, according to theorem 2.1. one can work equivalently with the system $(A \oplus 0, f \oplus \varphi)$, whose domain and range are vector spaces.

One can obviously define a category whose objects are of the type $(M, F,(a, f))$, where $M$ is a manifold, $F$ is a vector bundle over $M$ and $(a, f)$ is an IDE with domain $M$ and range $F$. A morphism $\varphi:(M, F,(a, f)) \rightarrow(N, G,(b, g))$ is given by a map $\varphi_{d}: M \rightarrow N$ 
and a vector bundle map $\varphi_{r}: F \rightarrow G$ over $\varphi_{d}$ such that, for any $(x, \dot{x}) \in M$, we have $\varphi_{r}(a(x) \dot{x})=b(y) \dot{y}$, and $g(y)=\varphi_{r}(f(x))$, where $(y, \dot{y})=T \varphi_{d}(x, \dot{x})$. However, although this perspective is interesting, we will not use the categorical context in this paper since, as we have said before, we want to keep the context as basic as possible.

Basic reduction algorithm for solving an IDE. We have the following basic algorithm to solve (2.1) recursively. It is a reformulation in our context of essentially known ideas contained in the references given before. However, we must remark that the fact that we have chosen to write a given IDE in the form (2.1) where the space (or more generally, vector bundle) $F$, remains unchanged (or is replaced by a pull-back bundle) throughout the reduction process, briefly described next, presents some clear technical advantages.

$\left(a_{1}\right) M_{1} \subseteq M$ is the subset of all $x \in M$ such that the LAS (2.1) has solution.

$\left(a_{2}\right)$ For $k=1,2, \ldots$ we assume that $M_{k}$ is a submanifold and $M_{k+1} \subseteq M_{k}$ is the subset of all $x \in M_{k}$ such that the LAS (2.1) has a solution $(x, \dot{x}) \in T M_{k}$.

If in a given example the assumptions that $M_{k}$ is a submanifold made at each stage of the previous algorithm are satisfied then the algorithm itself stabilizes at a certain stage $q$, that is $M_{q}=M_{q+1}$. Then the system (2.1) restricted to $M_{q}$ has a solution $(x, \dot{x}) \in T M_{q}$, as a LAS, for each $x \in M_{q}$. Thus we obtain an IDE with domain $M_{q}$ and range $F$ which may or may not be of constant rank according to the definition given before, but it is equivalent to the original one and the associated LAS has solution for all $x \in M_{q}$. The assumption that $M_{k+1} \subseteq M_{k}$ is a submanifold is obtained in practice by using, somehow, the subimmersion theorem or equivalent results. An important case in which this scheme works is the case where $M$ is a vector space, $a(x)$ is independent of $x$ and $f(x)$ is linear in $x$. This kind of situation was studied in 35. It can be also studied with our methods, leading to perfectly identifiable and simple desingularizing systems, which we are not going to explain in the present work.

\section{Desingularization}

The hypothesis that $M_{k}$ is a submanifold at each stage of the basic algorithm described above is too restrictive as it is not satisfied for many examples of interest. Some rather general geometric approaches to IDE in the $C^{\infty}$ category, like in [13, 18, also assume some kind of geometric version of the subimmersion theorem. We are going to show 
that in order to overcome part of those limitations, and at the cost of working in the subanalytic category rather than the $C^{\infty}$ category, one can use results from real analytic desingularization theory. This is possible thanks to the now well established theory of semianalytic and subanaliytic sets developed originally by Lojasiewicz [36, 37, 38. Important results in this field and systematic expositions using techniques which are simpler than the original ones are due to Gabrielov [39], Hironaka [29, Hardt [40, 41, Bierstone and Milman [30, Sussmann [32, and others. Our main reference will be 30, where an excellent and very readable exposition of important points of the theory of subanalytic sets has been written.

For the rest of this paper manifolds and maps will be real analytic, unless otherwise specified. For instance, if $(a, f)$ is a given IDE with domain $M$ then $M$ will be a real analytic manifold and $a, f$ will be real analytic maps.

Definition 3.1 Let $M$ be a real analytic manifold and let $X$ be a closed subanalytic subset of $M$. A desingularization of $X$ is a a real proper analytic map $f: N \rightarrow M$ such that $f(N)=X$, where $N$ is a real analytic manifold of the same dimension as $X$.

This is a relatively weak notion of desingularization, but it is enough for our purposes. Existence of desingularizations $f: N \rightarrow M$ is guaranteed by the following theorem of Hironaka, see [30, 32] and references therein.

Theorem 3.2 Let $M$ be a real analytic manifold and let $X$ be a closed subanalytic subset. Then there is a desingularization $f: N \rightarrow M$ of $X$.

Desingularization results that include those of Hironaka have been recently proved in Bierstone and Milman [27.

In fact, in the present paper we only need the following weaker desingularization result, which is theorem 5.1 in [30],

Theorem 3.3 Let $M$ be a real analytic manifold and let $X$ be a closed analytic subset. Then there is a desingularization $f: N \rightarrow M$ of $X$.

Description of the algorithm. Let $M$ be a manifold of dimension $d$ and let $(a, f)$ be a given IDE with domain $M$ and range $F$. The basic result proved in this paper to solve the IDE (2.1) consists, roughly, in transforming it into an equivalent IDE, say

$$
\tilde{a}_{2}(y) \dot{y}=\tilde{f}_{2}(y)
$$


on a manifold $\tilde{M}_{2}$, which is a DAS of constant rank. The manifold $\tilde{M}_{2}$ will be constructed by an algorithm that involves a desingularization process.

The decomposition $M=M_{0} \cup M_{1} \cup M_{2}$. First, let us assume that $M$ is a connected manifold of dimension $d$. For $i=0,1, \ldots$, let

$$
\begin{aligned}
S_{i}(M) & =\{x \in M \mid \operatorname{rank} a(x) \leq i\} \\
& =\{x \in M \mid \operatorname{det} A(x)=0, A(x) \text { submatrix of } a(x) \text { of order } i+1\}
\end{aligned}
$$

$S_{i}(M)$ is clearly a closed analytic subset of $M$, defined by analytic equations, for $i=$ $0,1, \ldots$

Also, for $i=0,1, \ldots$, let $L_{i}(M) \subseteq S_{i}(M)$ be defined by

$$
\begin{aligned}
L_{i}(M) & =\left\{x \in S_{i}(M) \mid \operatorname{rank}[a(x), f(x)] \leq i\right\} \\
& =\left\{x \in S_{i}(M) \mid \operatorname{det} A(x)=0, A(x) \text { submatrix of }[a(x), f(x)] \text { of order } i+1\right\} .
\end{aligned}
$$

Each $L_{i}(M)$ is a closed analytic subset of $M$ defined by analytic equations.

Let

$$
S_{k_{1}}(M) \subset S_{k_{2}}(M) \subset \ldots \subset S_{k_{r}}(M),
$$

where $k_{r}=k_{r}(M)$, be the distinct nonempty $S_{i}(M)$. We observe that $S_{k_{r}}(M) \equiv M$. Consider the corresponding inclusions

$$
L_{k_{1}}(M) \subseteq L_{k_{2}}(M) \subseteq \ldots \subseteq L_{k_{r}}(M) .
$$

We have that $\operatorname{rank} a(x)=\operatorname{rank}[a(x), f(x)]=k_{j}$ for each $x \in L_{k_{j}}(M)-S_{k_{j-1}}(M)$, $j=1, \ldots, r$. The LAS associated to (2.1) has solution for each $x \in L_{k_{j}}(M)-S_{k_{j-1}}(M)$, $j=1, \ldots, r$, where we have, by definition, $S_{k_{0}}=\emptyset$.

We remark the following useful facts: the set $L_{k_{j}}(M)-S_{k_{j}-1}(M)$ may be empty, for some $j=1, \ldots, r$; we have $\operatorname{dim} S_{k_{r-1}}(M)<\operatorname{dim} M$; if $\operatorname{dim}\left(L_{k_{r}}(M)\right)=d$, then $L_{k_{r}}(M)=M$.

Now let $M$ be a manifold of dimension $d$ and assume that

$$
M_{m}=\bigcup_{j} W_{j}
$$

is the union of the connected components of $M$ of maximal dimension $d$.

We will consider the following pairwise disjoint conditions for a given $W_{j} \subseteq M_{m}$, 
(a) $L_{k_{r}}\left(W_{j}\right)=\emptyset$

(b) $L_{k_{r}}\left(W_{j}\right) \neq \emptyset$ and $\operatorname{dim} L_{k_{r}}\left(W_{j}\right)<d$

(c) $L_{k_{r}}\left(W_{j}\right) \neq \emptyset$ and $\operatorname{dim} L_{k_{r}}\left(W_{j}\right)=d$.

We now define the following pairwise disjoint subsets of $M$.

$$
\begin{aligned}
& M_{0}=\left(M-M_{m}\right) \cup \bigcup_{b} L_{k_{r}}\left(W_{j}\right) \cup \bigcup_{c} S_{k_{r-1}}\left(W_{j}\right) \\
& M_{1}=\bigcup_{a} W_{j} \cup \bigcup_{b}\left(W_{j}-L_{k_{r}}\left(W_{j}\right)\right) \\
& M_{2}=\bigcup_{c}\left(W_{j}-S_{k_{r-1}}\left(W_{j}\right)\right) .
\end{aligned}
$$

We have the following assertions, whose proof is easy: each subset $L_{k_{r}}\left(W_{j}\right) \subseteq W_{j}$, and each subset $S_{k_{r-1}}\left(W_{j}\right) \subseteq W_{j}$, is a closed analytic subset of $W_{j}$ defined by analytic equations on $W_{j}$. In consequence, $W_{j}-L_{k_{r}}\left(W_{j}\right), W_{j}-S_{k_{r-1}}\left(W_{j}\right)$ are open submanifolds of $W_{j}$.

The manifold $M$ is the disjoint union

$$
M=M_{0} \cup M_{1} \cup M_{2} .
$$

The manifolds $M_{1}$ and $M_{2}$ are open submanifolds of $M$. The subset $M_{0}$ is a union of subsets defined by analytic equations on each $W_{j}$, union $M-M_{m}$, and we have that $\operatorname{dim} M_{0}<d$.

Restrictions $(a, f)\left|M_{0},(a, f)\right| M_{1},(a, f) \mid M_{2}$ and desingularization of $(a, f) \mid M_{0}$. We have that the LAS associated to (2.1) has no solution for $x \in M_{1}$. On the other hand, it has solution for all $x \in M_{2}$, moreover, $(a, f) \mid M_{2}$, is an IDE of constant rank.

It remains to see what happens with the system restricted to $M_{0}$. The idea here is to desingularize each closed analytic subset $L_{k_{r}}\left(W_{j}\right) \subseteq W_{j}$, and $S_{k_{r-1}}\left(W_{j}\right) \subseteq W_{j}$. By forming the disjoint union of those desingularizations and $M-M_{m}$ one obtains a desingularization of $M_{0}$ say

$$
\pi_{0}: M^{1} \rightarrow M, \text { where } \pi_{0}\left(M^{1}\right)=M_{0} .
$$

Then (2.1) restricted to $M_{0}$, that is $(a, f) \mid M_{0}$, can be naturally lifted, using the pullback operation, to an $\operatorname{IDE}\left(a_{1}, f_{1}\right)=\pi_{0}^{*}\left((a, f) \mid M_{0}\right)$ on $M^{1}$ as follows

$$
\begin{array}{r}
a_{1}(y) \dot{y}=a\left(\pi_{0}(y)\right) T_{y} \pi_{0}(y, \dot{y}) \\
f_{1}(y)=f\left(\pi_{0}(y)\right) .
\end{array}
$$


We should remark at this point (see also the paragraph Some notation and operations with IDE, in section 2) that in the present paper a tangent vector $(x, \dot{x})$ to $M_{0}$ at a point $x \in M_{0}$ is a vector $(x, \dot{x}) \in T_{x} M$ such there is an analytic curve $z(t) \in M_{0}$, say defined for $t \in(-\delta, \delta)$, such that $z(0)=x$ and the tangent vector to $z(t)$ at $t=0$ as a curve in $M$ coincides with $(x, \dot{x})$. In particular, if $y(t)$ is a given analytic curve in $M^{1}$ then

$$
T_{y} \pi_{0}(y, \dot{y})=\left.\frac{d \pi_{0}(y(t))}{d t}\right|_{t=0},
$$

is a tangent vector to $M_{0}$ at $\pi_{0}(y(0))$.

Note that $M^{1}$ is a manifold of dimension $\operatorname{dim} M^{1}=\operatorname{dim} M_{0}<d$.

Complete desingularization of $(a, f)$ in a finite number of steps. Now we repeat the process for the IDE $\left(a_{1}, f_{1}\right)$ with domain $M^{1}$ and range $F$, proceeding as we did before with the system $(a, f)$ with domain $M$ and range $F$. We obtain a decomposition

$$
M^{1}=M_{0}^{1} \cup M_{1}^{1} \cup M_{2}^{1} .
$$

We know that there is no solution to the LAS system

$$
a_{1}(y) \dot{y}=f_{1}(y)
$$

for $y \in M_{1}^{1}$. We also know that there is solution to the same LAS system for $y \in M_{2}^{1}$, moreover, $\left(a_{1}, f_{1}\right) \mid M_{2}^{1}$ is an IDE of constant rank. Now we desingularize $M_{0}^{1}$

$$
\pi_{1}: M^{2} \rightarrow M^{1}, \pi_{1}\left(M^{2}\right)=M_{0}^{1}
$$

and repeat the process. Finally, we obtain a finite sequence of manifolds and maps

$$
M^{q} \stackrel{\pi_{q-1}}{\rightarrow} M^{q-1} \stackrel{\pi_{q-2}}{\rightarrow} \ldots \stackrel{\pi_{1}}{\rightarrow} M^{1} \stackrel{\pi_{0}}{\rightarrow} M
$$

where $\pi_{0}\left(M^{1}\right)=M_{0}, \pi_{1}\left(M^{2}\right)=M_{0}^{1}$, and in general $\pi_{i}\left(M^{i+1}\right)=M_{0}^{i}$, for $i=0, \ldots, q-1$, where we have written $M^{0} \equiv M$ to unify the notation.

We have obtained a finite recursive procedure that reduces the problem to a finite number of IDE of constant rank, namely, the IDE of constant rank $\left(a_{i}, f_{i}\right) \mid M_{2}^{i}$, for $i=0,1, \ldots, q$, where we have written $\left(a_{0}, f_{0}\right)=(a, f)$, to unify the notation. We will call this a desingularization process and the sequence of maps $\pi_{i}$ and IDE $\left(a_{i+1}, f_{i+1}\right), i=0, \ldots, q-1$ a desingularization of $(a, f)$. 
The collection $\left(\tilde{a}_{2}, \tilde{f}_{2}\right)$ of IDE $\left(a_{k}, f_{k}\right) \mid M_{2}^{k}, k=0, \ldots, q$, defines a single IDE of constant rank in the disjoint union $\tilde{M}_{2}=\bigsqcup_{k=0}^{q} M_{2}^{k}$, as we have said at the beginning of the paragraph Description of the algorithm. We have a natural projection $\tilde{\pi}_{2}: \tilde{M}_{2} \rightarrow M$. This IDE $\left(\tilde{a}_{2}, \tilde{f}_{2}\right)$ with domain $\tilde{M}_{2}$ and range $F$ is called the desingularizing IDE.

Remark. As we have said before the range $F$ remains the same throughout the application of the algorithm. However, in practice it is sometimes convenient to apply theorem 2.1. which may imply a change of $F$, to simplify calculations.

\section{The Main Results}

In this section we will show in which precise sense the solutions to the desingularizing system $\left(\tilde{a}_{2}, \tilde{f}_{2}\right)$ of a given analytic IDE $(a, f)$ are related to the solutions to $(a, f)$. It is clear that in certain simple examples of IDE one can show in a more or less direct way that solutions to the desingularizing system project via $\tilde{\pi}_{2}$ onto solutions to the given IDE, and also that solutions to the IDE are such projections. For instance, if one is interested in the local behavior of solutions near a singular point of $M_{0}$ it is sometimes enough to use a simple blow-up to desingularize $M_{0}$ at that point, and one can show in certain cases in a simple and useful way the relationship between solutions to the given system and solutions to the system obtained by blow-up, see 4. However, in this paper we want a more precise and general global result showing that a curve, belonging to a certain convenient class of curves, is a solution to a given IDE if and only if it is essentially the projection via the map $\tilde{\pi}_{2}$ of a solution to the desingularizing system belonging to the same class of curves. This is important, for instance, if one is interested in global aspects of solutions, like extension of solutions, or in a description of the family of all solutions. In order to be able to use the theory of subanalytic sets we need to define carefully a convenient class of curves, which we will do in the next paragraph.

From now on, we will often use the theorem 6.1 of 30] that a subanalytic subset of dimension 1 of an analytic manifold $M$ is a semianalytic subset. We will also often use the fact that the image of a relatively compact subanalytic subset under a subanalytic map is a subanalytic subset, see [30. immediately after definition 3.2. Using this we can deduce that the image of a relatively compact subanalytic subset under an analytic map is a subanalytic subset, which will be also useful for us.

In what follows, we will work with several types of intervals, like $\left(\tau_{0}, \tau_{1}\right),\left[\tau_{0}, \tau_{1}\right),\left(\tau_{0}, \tau_{1}\right]$ or $\left[\tau_{0}, \tau_{1}\right]$. We will usually assume that $\tau_{0}$ and $\tau_{1}$ are real numbers. However, some definitions and results are valid also for the case in which the open end of an interval is 
$\pm \infty$, that is, for intervals $\left(-\infty, \tau_{1}\right) ;\left(\tau_{0},+\infty\right) ;(-\infty,+\infty) ;\left[\tau_{0},+\infty\right) ;\left(-\infty, \tau_{1}\right]$

The notion of an as-curve. Inspired by [30, definition 3.2, we will define

Definition 4.1 A subanalytic curve $x:\left(t_{0}, t_{1}\right) \rightarrow M$ is a subanalytic map, that is, a map such that graph $x \subseteq \mathbb{R} \times M$ is a subanalytic subset. We define the notion of a subanalytic curve $x:\left[t_{0}, t_{1}\right) \rightarrow M, x:\left(t_{0}, t_{1}\right] \rightarrow M$ or $x:\left[t_{0}, t_{1}\right] \rightarrow M$ in a similar way.

In definition 4.1 since $\operatorname{dim}(\operatorname{graph} x)=1$ we have that graph $x$ is a semianalytic set.

Lemma $4.2(a)$ Let $x:\left[t_{0}, t_{1}\right) \rightarrow M$ be a continuous subanalytic curve whose graph is a relatively compact subset. Then there is a uniquely defined continuous subanalytic extension $\bar{x}:\left[t_{0}, t_{1}\right] \rightarrow M$. A similar result holds for subanalytic curves $x:\left(t_{0}, t_{1}\right] \rightarrow M$ or $x:\left(t_{0}, t_{1}\right) \rightarrow M$.

(b) Let $x:\left[t_{0}, t_{1}\right) \rightarrow M$ be a continuous subanalytic curve whose graph is not a relatively compact subset. Then graph $x$ is closed. A similar result holds for subanalytic curves $x:\left(t_{0}, t_{1}\right] \rightarrow M$ or $x:\left(t_{0}, t_{1}\right) \rightarrow M$.

Proof. First we shall prove $(a)$. We need to show first that the limit of $x(t)$ as $t \rightarrow t_{1}^{-}$exists. Using corollary 2.8 of 30 we can deduce that the closure $\bar{G}$ of $G=\operatorname{graph} x$ in $\mathbb{R} \times M$ is subanalytic and compact, and then also $\bar{G} \cap\left(\left\{t_{1}\right\} \times M\right)$ is subanalytic and compact. Since $\bar{G} \cap\left(\left\{t_{1}\right\} \times M\right)$ is nonempty let $x_{1} \in \bar{G} \cap\left(\left\{t_{1}\right\} \times M\right)$ be given. One can choose local coordinates at $x_{1}$, and for any small $\epsilon>0$ the set $G_{\epsilon}=G \cap\left(\left(t_{0}, t_{1}\right) \times B_{\epsilon}\left(x_{1}\right)\right)$ is relatively compact. We can easily deduce from theorem 3.14 of 30 that any relatively compact subanalytic set has a finite number of connected components. Let $C_{i}, i=1, \ldots, n(\epsilon)$ be the connected components of $G_{\epsilon}$. It is not difficult to see that each connected component $C_{i}$ is of the type $C_{i}=\operatorname{graph}\left(x \mid\left(\alpha_{i}, \beta_{i}\right)\right), i=1, \ldots, n(\epsilon)$. We can assume without loss of generality that $\beta_{i} \leq \alpha_{i+1}, i=1, \ldots, n(\epsilon)-1$. Since $x_{1}$ is a limit point of $G_{\epsilon}$ we must have $\beta_{n(\epsilon)}=t_{1}$, which implies that $x(t) \in B_{\epsilon}\left(x_{1}\right)$ for all $t \in\left(\alpha_{n(\epsilon)}, \beta_{n(\epsilon)}\right)$, as we wanted to prove. The fact that the continuous extension $\bar{x}:\left[t_{0}, t_{1}\right] \rightarrow M$ is a subanalytic curve follows from corollary 2.8 of [30. The rest of the proof of $(a)$ can be performed in a similar way. Now we will prove $(b)$. If graph $x$ is not relatively compact then $\bar{G} \cap\left(\left\{t_{1}\right\} \times M\right)$ must be empty, otherwise we can proceed as in the proof of $(a)$ and we can conclude that the limit of $x(t)$ as $t \rightarrow x_{1}^{-}$exists and then, one can show that $G$ is relatively compact. Since all the limit points of $G$ not in $G$ must belong to $\bar{G} \cap\left(\left\{t_{1}\right\} \times M\right)$ we have that $G$ is closed. The rest of the proof of $(b)$ can be performed in a similar way.

In order to define a convenient class of curves to solve a given IDE we introduce the following notion 
Definition 4.3 (a) An analytic-semianalytic-curve $x(t), t \in\left(t_{0}, t_{1}\right)$, in $M$, where $M$ is a given manifold, is an analytic map $x:\left(t_{0}, t_{1}\right) \rightarrow M$ which is also a subanalytic curve, that is, such that graph $x$ is a semianalytic subset of $\mathbb{R} \times M$. We will often call such an analytic-semianalytic-curve in $M$ simply an as-curve in $M$.

(b) An analytic-semianalytic-curve (or as-curve) $x(t), t \in\left[t_{0}, t_{1}\right),\left(t \in\left(t_{0}, t_{1}\right], t \in\left[t_{0}, t_{1}\right]\right)$ in $M$, where $M$ is a given manifold, is a continuous map $x:\left[t_{0}, t_{1}\right) \rightarrow M$, (respectively, $\left.x:\left(t_{0}, t_{1}\right] \rightarrow M, x:\left[t_{0}, t_{1}\right] \rightarrow M\right)$, which is also a subanalytic curve, that is, such that graph $x$ is a semianalytic subset of $\mathbb{R} \times M$, and $x \mid\left(t_{0}, t_{1}\right)$ is an as-curve in $M$.

For instance, $x=\sqrt{t}, t \in(0, c)$, (or $t \in[0, c), t \in(0, c], t \in[0, c])$, with $c>0$, are as-curves in $\mathbb{R}$. On the other hand, $x=t \sin (\pi / t), t \in(0, c)$, with $c>0$, is not an as-curve in $\mathbb{R}$, but $x=t \sin (\pi / t), t \in(\delta, c)$, with $0<\delta<c$, is an as-curve in $\mathbb{R}$.

Next, we will give some lemmas where some basic properties of as-curves, that we need to prove our main results, are proved.

Lemma $4.4(a)$ Let $x:\left(t_{0}, t_{1}\right) \rightarrow N$ be a given analytic map, where $N$ is a given manifold. Then any map $x \mid\left(\bar{t}_{0}, \bar{t}_{1}\right):\left(\bar{t}_{0}, \bar{t}_{1}\right) \rightarrow N$, where $\bar{t}_{0}$ and $\bar{t}_{1}$ are such that $t_{0}<$ $\bar{t}_{0}<\bar{t}_{1}<t_{1}$, is an as-curve in $N$. In a similar way, any map $x \mid\left[\bar{t}_{0}, \bar{t}_{1}\right):\left[\bar{t}_{0}, \bar{t}_{1}\right) \rightarrow N$, $x \mid\left(\bar{t}_{0}, \bar{t}_{1}\right]:\left(\bar{t}_{0}, \bar{t}_{1}\right] \rightarrow N$, or $x \mid\left[\bar{t}_{0}, \bar{t}_{1}\right]:\left[\bar{t}_{0}, \bar{t}_{1}\right] \rightarrow N$, with $\bar{t}_{0}$ and $\bar{t}_{1}$ as before is an as-curve in $N$.

(b) Let $x:\left(t_{0}, t_{1}\right) \rightarrow N$ be an as-curve and assume that graph $x$ is a relatively compact subset of $\mathbb{R} \times N$. Then there is a unique continuous extension $\bar{x}:\left[t_{0}, t_{1}\right] \rightarrow M$ which is an as-curve.

Proof. Let us prove $(a)$. We have that graph $\left(x \mid\left(\bar{t}_{0}, \bar{t}_{1}\right)\right)$ is a semianalytic subset of $\mathbb{R} \times N$ defined as $\left\{(t, x) \in\left(t_{0}, t_{1}\right) \times N: \bar{t}_{0}<t<\bar{t}_{1}, x=x(t)\right\}$. The rest $(a)$ can be proved in a similar way. To prove $(b)$ we simply apply lemma 4.2

Lemma 4.5 Let $x:\left[t_{0}, t_{1}\right) \rightarrow M$ be a subanalytic map and assume that $x$ is continuous at $t_{0}$. Then there exists $t_{2} \in\left(t_{0}, t_{1}\right)$ such that $x \mid\left[t_{0}, t_{2}\right]$ is an as-curve. A similar result holds for a subanalytic map $x:\left(t_{0}, t_{1}\right] \rightarrow M$ continuous at $t_{1}$, that is, there exists $t_{2} \in\left(t_{0}, t_{1}\right)$ such that $x \mid\left[t_{2}, t_{1}\right]$ is an as-curve.

Proof. If $x$ is a constant the result follows immediately. Let us assume that $x$ is not a constant. It is easy to see that $\operatorname{graph}\left(x \mid\left[t_{0}, t_{2}\right]\right)$ is a semianalytic subset of $\mathbb{R} \times M$ of dimension 1 , for every $t_{2} \in\left(t_{0}, t_{1}\right)$. We can assume without loss of generality, using, for instance, Whitney embedding theorem, that $M \subseteq U$ is an analytic submanifold of $U$, where $U$ is a real finite dimensional vector space. Let $p_{1}: \mathbb{R} \times U \rightarrow \mathbb{R}$ be the projection onto the first factor. Continuity of $x$ at $t_{0}$ implies that one can choose $b \in\left(t_{0}, t_{1}\right)$, such that $\operatorname{graph}\left(x \mid\left[t_{0}, b\right]\right)$ is a relatively compact subanalytic subset of $\mathbb{R} \times U$. According to 
lemma 3.4 of [30] we have that $\operatorname{graph}\left(x \mid\left[t_{0}, b\right]\right)$ is a finite union of connected smooth semianalytic subsets $A$ such that, for each $A, \operatorname{rank}\left(p_{1} \mid A\right)$ is constant. It is not difficult to see that each $A$ has dimension 0 or 1 and that $\operatorname{rank}\left(p_{1} \mid A\right)$ is 0 or 1 . Moreover, we can see that there must be an $A$, say $A=A_{0}$, such that $\operatorname{rank}\left(p_{1} \mid A_{0}\right)=1, p_{1}\left(A_{0}\right)=\left(t_{0}, t_{2}\right)$, for some $t_{2} \in\left(t_{0}, b\right]$, and $p_{1}\left(\bar{A}_{0}\right)=\left[t_{0}, t_{2}\right]$. From this we can easily deduce that $x \mid\left(t_{0}, t_{2}\right)$ is an as-curve and moreover, using lema 4.4. (b), that $x \mid\left[t_{0}, t_{2}\right]$ is an as-curve. The rest of the proof can be performed in a similar way.

Lemma 4.6 (a) Let $x:\left[t_{0}, t_{1}\right] \rightarrow M, x:\left[t_{0}, t_{1}\right) \rightarrow M$ or $x:\left(t_{0}, t_{1}\right] \rightarrow M$ be an as-curve in $M$. Then $x \mid\left(t_{0}, t_{1}\right)$ is an as-curve in $M$.

(b) Let $x:\left(t_{0}, t_{1}\right) \rightarrow M$ be an as-curve in $M$ and assume that there is a continuous extension $\bar{x}:\left[t_{0}, t_{1}\right] \rightarrow M, \bar{x}:\left[t_{0}, t_{1}\right) \rightarrow M$ or $\bar{x}:\left(t_{0}, t_{1}\right] \rightarrow M$. Then $\bar{x}$ is an as-curve in $M$.

(c) Let $f: M \rightarrow N$ be a given analytic map. Let $x(t), t \in\left[t_{0}, t_{1}\right)\left(t \in\left(t_{0}, t_{1}\right], t \in\left[t_{0}, t_{1}\right]\right.$, $\left.t \in\left(t_{0}, t_{1}\right)\right)$ be an as-curve in $M$. Then $f(x(t)), t \in\left[\bar{t}_{0}, \bar{t}_{1}\right]$, is an as-curve in $N$, for each $\left[\bar{t}_{0}, \bar{t}_{1}\right] \subseteq\left[t_{0}, t_{1}\right)$, (respectively, $\left.\left[\bar{t}_{0}, \bar{t}_{1}\right] \subseteq\left(t_{0}, t_{1}\right],\left[\bar{t}_{0}, \bar{t}_{1}\right] \subseteq\left[t_{0}, t_{1}\right],\left[\bar{t}_{0}, \bar{t}_{1}\right] \subseteq\left(t_{0}, t_{1}\right)\right)$. If graph $x$ is relatively compact then $f(x(t)), t \in\left[t_{0}, t_{1}\right),\left(t \in\left(t_{0}, t_{1}\right], t \in\left[t_{0}, t_{1}\right], t \in\left(t_{0}, t_{1}\right)\right)$ is an as-curve and $\operatorname{graph}(x \circ f)$ is relatively compact.

Proof. Part $(a)$ is an immediate consequence of the definitions. Part $(b)$ follows easily using corollary 2.8 of 30 . To prove $(c)$ observe first that $f \circ x:\left(t_{0}, t_{1}\right) \rightarrow N$ is an analytic map. Since graph $\left(x \mid\left[\bar{t}_{0}, \bar{t}_{1}\right]\right)$ is a semianalytic compact subset of $\mathbb{R} \times M$ of dimension 1 we have that $\operatorname{graph}\left(f \circ x \mid\left[\bar{t}_{0}, \bar{t}_{1}\right]\right)=\left(1_{\mathbb{R}} \times f\right)\left(\operatorname{graph}\left(x \mid\left[\bar{t}_{0}, \bar{t}_{1}\right]\right)\right.$, taking into account that $1_{\mathbb{R}} \times f: \mathbb{R} \times M \rightarrow \mathbb{R} \times N$ is an analytic map, is also semianalytic, and a similar proof can be given for the case of the intervals $\left(t_{0}, t_{1}\right],\left[t_{0}, t_{1}\right],\left(t_{0}, t_{1}\right)$. If graph $x$ is relatively compact then according to lemma 4.2 we have a continuous extension $\bar{x}$ which is an as-curve and therefore we can apply the first part of $(c)$ to this extension The rest of the proof follows easily.

Lemma 4.7 Let $N$ be a given manifold and let $x:\left[t_{0}, t_{1}\right) \rightarrow N$ be an as-curve in $N$ which is not a constant. Then there exist $t_{2} \in\left(t_{0}, t_{1}\right)$ such that $x\left(\left(t_{0}, t_{2}\right)\right)$ is an analytic submanifold which is also a semianalytic subset, $x \mid\left(t_{0}, t_{2}\right):\left(t_{0}, t_{2}\right) \rightarrow x\left(\left(t_{0}, t_{2}\right)\right)$ is an analytic diffeomorphism and $x \mid\left[t_{0}, t_{2}\right]:\left[t_{0}, t_{2}\right] \rightarrow x\left(\left[t_{0}, t_{1}\right]\right)$ is an homeomorphism. Similar results hold for an as-curve $x:\left(t_{0}, t_{1}\right] \rightarrow N$.

Proof. Let $t_{0}<\bar{t}_{1}<t_{1}$, then we have that $\operatorname{graph}\left(x \mid\left[t_{0}, \bar{t}_{1}\right]\right)$ is a compact semianalytic subset of $\mathbb{R} \times N$ of dimension 1 . Let $x_{i}, i=1, \ldots, n$ be local analytic coordinates centered at $x\left(t_{0}\right)$ therefore $x_{i}\left(t_{0}\right)=0$ for $i=1, . ., n$. Without loss of generality we can assume that 
$x_{i}(t)$ is defined for all $i=1, . ., n$ and all $t \in\left[t_{0}, \bar{t}_{1}\right]$. For some index, say $j \in\{1, \ldots, n\}$, we must have that $x_{j}(t)$ is not a constant. We are going to show that there exists $t_{2} \in\left(t_{0}, \bar{t}_{1}\right]$ such that $x_{j}:\left[t_{0}, t_{2}\right] \rightarrow \mathbb{R}$ satisfies certain conditions from which the lemma follows. Since $x_{j}(t)$ is the projection on the $j$-coordinate axis of the curve $x \mid\left[t_{0}, \bar{t}_{1}\right]$ using lemma 4.6] (c) we have that graph $\left(x_{j} \mid\left[t_{0}, \bar{t}_{1}\right]\right) \subseteq \mathbb{R} \times \mathbb{R}$ is a semianalytic compact subset of dimension 1 . The restriction to graph $\left(x_{j} \mid\left[t_{0}, \bar{t}_{1}\right]\right)$ of the projection $p_{2}: \mathbb{R} \times \mathbb{R} \rightarrow \mathbb{R}$ onto the second factor satisfies $p_{2}\left(t, x_{j}(t)\right)=x_{j}(t)$, for all $t \in\left[t_{0}, \bar{t}_{1}\right]$. Then according to lemma 3.4 of [30] graph $\left(x_{j} \mid\left[t_{0}, \bar{t}_{1}\right]\right)$ is a finite union of connected smooth semianalytic subsets $A$ such that $\operatorname{rank}\left(p_{2} \mid A\right)$ is constant on $A$, and it is easy to show that $\operatorname{rank}\left(p_{2} \mid A\right)$ can take only the values 0 or 1 . It is also easy to see that for at least one such $A$ one must have that $\operatorname{rank}\left(p_{2} \mid A\right)=1, p_{2}(A)=x_{j}\left(\left(t_{0}, t_{2}\right)\right)$ for some $t_{2} \in\left(t_{0}, \bar{t}_{1}\right]$ and $\overline{p_{2}(A)}=p_{2}(\bar{A})=$ $x_{j}\left(\left[t_{0}, t_{2}\right]\right)$, and therefore that $x_{j}\left(\left(t_{0}, t_{2}\right)\right)$ is an open interval. Moreover $x_{j} \mid\left[t_{0}, t_{2}\right]$ is injective. and we have that $\left(x_{j} \mid\left(t_{0}, t_{2}\right)\right)^{-1}: x_{j}\left(\left(t_{0}, t_{2}\right)\right) \rightarrow\left(t_{0}, t_{2}\right)$ is analytic and also its graph is a semianalytic subset of $\mathbb{R} \times \mathbb{R}$ and then, because of lemma 4.4, (b), that there is an extension $\left(x_{j} \mid\left[t_{0}, t_{2}\right]\right)^{-1}: x_{j}\left(\left[t_{0}, t_{2}\right]\right) \rightarrow\left[t_{0}, t_{2}\right]$ which is continuous, and therefore $x_{j} \mid\left[t_{0}, t_{2}\right]:\left[t_{0}, t_{2}\right] \rightarrow x_{j}\left(\left[t_{0}, t_{1}\right]\right)$ is an homeomorphism. Let $t(s), s \in\left[x_{j}\left(t_{0}\right), x_{j}\left(t_{2}\right)\right]$, be the map $\left(x \mid\left[t_{0}, t_{2}\right]\right)_{j}^{-1}$, in other words, the parameter $s$ represents the coordinate $x_{j}$. Then we have that $x\left(\left[t_{0}, t_{2}\right]\right)=\left\{\left(x_{1}, \ldots, x_{n}\right): x_{i}=x_{i}(t(s)), s \in\left[x_{j}\left(t_{0}\right), x_{j}\left(t_{1}\right)\right], i=1, \ldots, n\right\}$ and also $x\left(\left(t_{0}, t_{2}\right)\right)=\left\{\left(x_{1}, \ldots, x_{n}\right): x_{i}=x_{i}(t(s)), s \in\left(x_{j}\left(t_{0}\right), x_{j}\left(t_{1}\right)\right), i=1, \ldots, n\right\}$. ¿From this we can easily deduce the assertion of the lemma for the case of an as-curve $x$ : $\left[t_{0}, t_{1}\right) \rightarrow N$. The case of an as-curve $x:\left(t_{0}, t_{1}\right] \rightarrow N$ can be proven in an entirely similar way.

Lemma 4.8 Let $x(t), t \in\left[t_{0}, t_{1}\right)$ be an as-curve in $N$, which is not a constant. Then there is a $t_{2} \in\left(t_{0}, t_{1}\right)$ such that $x\left(\left[t_{0}, t_{2}\right)\right)-\left\{x\left(t_{0}\right)\right\}$ is nonempty and locally connected at $x\left(t_{0}\right)$, more precisely, $x\left(\left(t_{0}, t_{3}\right)\right)$ is a neighborhood of $x\left(t_{0}\right)$ in $x\left(\left[t_{0}, t_{2}\right)\right)-\left\{x\left(t_{0}\right)\right\}$, for all $t_{3} \in\left(t_{0}, t_{2}\right)$. Moreover, $x:\left[t_{0}, t_{2}\right] \rightarrow x\left(\left[t_{0}, t_{2}\right]\right)$ is an homeomorphism, $x\left(\left(t_{0}, t_{2}\right)\right)$ is an analytic submanifold which is a semianalytic subset of $N$ and $x:\left(t_{0}, t_{2}\right) \rightarrow x\left(\left(t_{0}, t_{2}\right)\right)$ is an analytic diffeomorphism. A similar result holds for an as-curve $x(t), t \in\left(t_{0}, t_{1}\right]$, in $M$.

Proof. We can show using lemma 4.7 that there exists $t_{2} \in\left(t_{0}, t_{1}\right)$ such that $x \mid\left[t_{0}, t_{2}\right]$ is injective, and that $x \mid\left[t_{0}, t_{2}\right]$ is an homeomorphism onto its image and that $x:\left(t_{0}, t_{2}\right) \rightarrow$ $x\left(\left(t_{0}, t_{2}\right)\right)$ is an analytic diffeomorphism where $x\left(\left(t_{0}, t_{2}\right)\right)$ is an analytic submanifold. In particular, we have that $x(t) \neq x\left(t_{0}\right)$ for all $t \in\left(t_{0}, t_{2}\right]$. Let $r>0$ small be given. Working in local analytic coordinates centered at $x\left(t_{0}\right)$ we can show that continuity of $x(t)$ implies that there exists $t_{r} \in\left(t_{0}, t_{2}\right]$ such that $x\left(\left[t_{0}, t_{r}\right]\right) \subseteq B_{r}\left(x\left(t_{0}\right)\right)$. It can be easily shown that $x\left(\left[t_{0}, t_{r}\right)\right)-\left\{x\left(t_{0}\right)\right\}=x\left(\left(t_{0}, t_{r}\right)\right)$ is connected. Moreover, for each $s \in\left[t_{0}, t_{r}\right)$ there 
is an open ball $B_{\delta}(x(s)) \subseteq B_{r}\left(x\left(t_{0}\right)\right)$, with $\delta=\delta(s)$, such that $B_{\delta}(x(s)) \cap x\left(\left[t_{r}, t_{2}\right]\right)=\emptyset$. Then the open set

$$
W=\bigcup_{s \in\left[t_{0}, t_{r}\right)} B_{\delta}(x(s))
$$

satisfies $W \subseteq B_{r}\left(x\left(t_{0}\right)\right)$ and $W \cap\left(x\left(\left[t_{0}, t_{2}\right)\right)-\left\{x\left(t_{0}\right)\right\}\right)=x\left(\left(t_{0}, t_{r}\right)\right)$. This shows that $x\left(\left[t_{0}, t_{2}\right)\right)-\left\{x\left(t_{0}\right)\right\}$ is nonempty and locally connected at $x\left(t_{0}\right)$ and also that $x\left(\left(t_{0}, t_{r}\right)\right)$ is a neighborhood of $x\left(t_{0}\right)$ in $x\left(\left[t_{0}, t_{2}\right)\right)-\left\{x\left(t_{0}\right)\right\}$. Now for each $t_{3} \in\left(t_{0}, t_{r}\right]$ take

$$
W_{t_{3}}=\bigcup_{s \in\left[t_{0}, t_{3}\right)} B_{\bar{\delta}}(x(s))
$$

where $\bar{\delta}=\bar{\delta}\left(t_{3}, s\right)$ and $B_{\bar{\delta}}(x(s))$ satisfies $B_{\bar{\delta}}(x(s)) \subseteq B_{r}(x(0))$ and $x\left(\left[t_{3}, t_{2}\right]\right) \cap B_{\bar{\delta}}(x(s))=$ $\emptyset$. Then $x\left(\left(t_{0}, t_{3}\right)\right)=W_{t_{3}} \cap\left(x\left(\left[t_{0}, t_{2}\right)\right)-\left\{x\left(t_{0}\right)\right\}\right)$. This shows that $x\left(\left(t_{0}, t_{3}\right)\right)$ is a neighborhood of $x\left(t_{0}\right)$ in $x\left(\left[t_{0}, t_{2}\right)\right)-\left\{x\left(t_{0}\right)\right\}$. Then the first case of the lemma is proved. The case of an as-curve $x(t), t \in\left(t_{0}, t_{1}\right]$, in $M$ can be proven in an entirely similar way.

Inspired by lemma 6.3 of [30] we will prove the following result about the image and reparametrization of an as-curve.

Lemma 4.9 Let $x(t) \in M, t \in\left[t_{0}, t_{1}\right)$, be an as-curve in $M$. Then there is an ascurve $z(s), s \in\left(s_{0}-\delta_{1}, s_{0}+\delta_{2}\right)$, in $M$, for some $\delta_{1}, \delta_{2}>0$ such that $z\left(s_{0}\right)=x\left(t_{0}\right)$, $z\left(\left[s_{0}, s_{0}+\delta_{2}\right)\right)=x\left(\left[t_{0}, t_{2}\right)\right)$ for some $t_{2} \in\left(0, t_{1}\right)$ and we also have that $t_{2}$ and $s_{0}+\delta_{2}$ are such that $x \mid\left[t_{0}, t_{2}\right)$ and $z \mid\left[s_{0}, s_{0}+\delta_{2}\right)$ are homeomorphisms onto $z\left(\left[s_{0}, s_{0}+\delta_{2}\right)\right)=x\left(\left[t_{0}, t_{2}\right)\right)$, $x \mid\left(t_{0}, t_{2}\right)$ and $z \mid\left(s_{0}, s_{0}+\delta_{2}\right)$ are analytic difeomorphisms onto $z\left(\left(s_{0}, s_{0}+\delta_{2}\right)\right)=x\left(\left(t_{0}, t_{2}\right)\right)$ which is an analytic submanifold which is also a semianalytic subset and, moreover, $\left(x \mid\left(t_{0}, t_{2}\right)\right)^{-1} \circ\left(z \mid\left(s_{0}, s_{0}+\delta_{2}\right)\right):\left(s_{0}, s_{0}+\delta_{2}\right) \rightarrow \mathbb{R}$ is an as-curve in $\mathbb{R}$ which is an analytic diffeomorfism onto its image $\left(t_{0}, t_{2}\right)$. Moreover, $t_{2}$ can be chosen such that for each $t_{3} \in\left(t_{0}, t_{2}\right], x\left(\left(t_{0}, t_{3}\right)\right)$ is a neighborhood of $x\left(t_{0}\right)$ in $x\left(\left[t_{0}, t_{2}\right)\right)-\left\{x\left(t_{0}\right)\right\}$. Similar results hold for as-curves $x(t)$ in $M$, where $t \in\left(t_{0}, t_{1}\right]$.

Proof. Using lemma 4.8 and also lemma 6.3 of [30, we can conclude that there is an as-curve $z(s), s \in\left(s_{0}-\delta_{1}, s_{0}+\delta_{1}\right)$, in $M$, for some $\delta_{1}, \delta_{2}>0$ such that $z\left(s_{0}\right)=x\left(t_{0}\right)$, $z\left(\left[s_{0}, s_{0}+\delta_{2}\right)\right)=x\left(\left[t_{0}, t_{2}\right)\right)$ for some $t_{2} \in\left(0, t_{1}\right)$ satisfying all the conditions stated in lemma 4.8. Since we can also apply lemma 4.5 and lemma 4.8 to $z(s)$ we can also deduce that $t_{2}$ and $\delta_{2}$ can be chosen such that $x \mid\left[t_{0}, t_{2}\right)$ and $z \mid\left[s_{0}, s_{0}+\delta_{2}\right)$ are homeomorphisms onto $z\left(\left[s_{0}, s_{0}+\delta_{2}\right)\right)=x\left(\left[t_{0}, t_{2}\right)\right), x \mid\left(t_{0}, t_{2}\right)$ and $z \mid\left(s_{0}, s_{0}+\delta_{2}\right)$ are analytic diffeomorphisms onto $z\left(\left(s_{0}, s_{0}+\delta_{2}\right)\right)=x\left(\left(t_{0}, t_{2}\right)\right)$ which is an analytic submanifold which is also a semianalytic subset and, moreover, $\left(z \mid\left(t_{0}, t_{3}\right)\right)^{-1} \circ\left(x \mid\left(t_{0}, t_{2}\right)\right):\left(t_{0}, t_{2}\right) \rightarrow \mathbb{R}$ is an as-curve in $\mathbb{R}$ which is an analytic diffeomorfism onto its image $\left(s_{0}, s_{0}+\delta_{2}\right)$. The rest of the proof 
can be performed in a similar way.

The notion of a pas-curve and of a lcs-curve. By definition, a continuous curve in a manifold $M, x(t), t \in[a, b)$ such that there is a partition $a=t_{0} \leq, \ldots, \leq t_{m}=b$ such that the restrictions $x\left|\left[t_{i}, t_{i+1}\right], i=0, \ldots, m-2, x\right|\left[t_{m-1}, t_{m}\right)$ are as-curves in $M$ is called a continuous piecewise-as-curve in $M$ (or continuous pas-curve in $M$ ). Each restriction $x\left|\left[t_{i}, t_{i+1}\right), x\right|\left(t_{i}, t_{i+1}\right], x\left|\left(t_{i}, t_{i+1}\right), x\right|\left[t_{i}, t_{i+1}\right], i=0, \ldots, m-2, x\left|\left[t_{m-1}, b\right), x\right|\left(t_{m-1}, b\right)$ is called an as-piece of $x$. We define the notion of a continuous piecewise-as-curve (or continuous pas-curve) $x(t), t \in(a, b], t \in[a, b]$ or $t \in(a, b)$, in $M$, in a similar way.

We shall also introduce the notion of a (not necessarily continuous) piecewise-as-curve in $M$ (or pas-curve in $M$ ) by eliminating from the previous definition the condition of continuity at the points $x\left(t_{i}\right), i=1, \ldots, m-1$, belonging to two consecutive as-pieces, and replacing it by the weaker condition of left or right continuity. More precisely, a pas-curve $x:[a, b) \rightarrow M$ in $M$ is defined by the condition that there is a partition $a=t_{0} \leq, \ldots, \leq t_{m}=b$ such that, for each $i=0, \ldots, m-2$ such that $t_{i} \neq t_{i+1}$, the restriction $x \mid\left(t_{i}, t_{i+1}\right)$ is an as-curve in $M$ having compact graph and $x \mid\left(t_{m-1}, b\right)$ is an as-curve in $M$ having a continuous extension $x \mid\left[t_{m-1}^{+}, b\right)$ obtained by taking the limit at $t_{m-1}$ on the right. Besides, we require that $x$ be left continuous or right continuous at each $t_{i}, i=1, \ldots, m-1$. We define the notion of a piecewise-as-curve (or pas-curve) $x(t)$, $t \in(a, b], t \in[a, b]$ or $t \in(a, b)$, in $M$, in a similar way.

We see immediately from this definition that any as-curve is a pas-curve and, moreover, that any continuous pas-curve is a pas-curve, in a natural way. Using lemma 4.2 we can conclude that, given a pas-curve $x:[a, b) \rightarrow M$ as before, for each $i=0, \ldots, m-2$ there is a well defined as-curve denoted $x \mid\left[t_{i}^{+}, t_{i+1}^{-}\right]$, which, for each $i=0, \ldots, m-2$ such that $t_{i} \neq t_{i+1}$, is the uniquely defined continuous extension of the restriction $x \mid\left(t_{i}, t_{i+1}\right)$. We can easily see from lemma 4.2 that if the graph of $x \mid\left[t_{m-1}^{+}, b\right)$ is not relatively compact then it must be closed. Similar statements hold for pas-curves $x(t), t \in(a, b], t \in[a, b]$ or $t \in(a, b)$, in $M$.

Given a pas-curve $x(t), t \in[a, b)$, as above, we have that some of the restrictions $x\left|\left[t_{i}, t_{i+1}\right), x\right|\left(t_{i}, t_{i+1}\right], x\left|\left[t_{i}, t_{i+1}\right], x\right|\left(t_{i}, t_{i+1}\right)$, for each $i=0, \ldots, m-2$, and some of the restrictions $x\left|\left[t_{m-1}, b\right)\right|\left(t_{m-1}, b\right)$, satisfy the required continuity condition at the closed end of the interval and then they are as-curves. Each one of those restrictions which is an as-curve is called an as-piece of $x$. If the graph of $x$ is relatively compact we will call each 
as-curve $x\left|\left[t_{i}^{+}, t_{i+1}^{-}\right], i=0, \ldots, m-2, x\right|\left[t_{m-1}^{+}, b^{-}\right]$an as-piece closure of $x$. If graph $x$ is not relatively compact then the as-piece closures are $x\left|\left[t_{i}^{+}, t_{i+1}^{-}\right], i=0, \ldots, m-2, x\right|\left[t_{m-1}^{+}, b\right)$. We must observe that an as-piece closure is an as-curve but it is not always an as-piece of $x$. By definition, the open as-pieces are $x\left|\left(t_{i}, t_{i+1}\right), i=0, \ldots, m-2, x\right|\left(t_{m-1}, b\right)$. Similar definitions hold for curves $x(t)$, where $t \in(a, b], t \in[a, b], t \in(a, b)$.

We need the following definition. A pas-curve $y$ in $M$ is a refinement of a pas-curve $z$ in $M$ if graph $y=\operatorname{graph} z$ and the graph of each as-piece of $y$ is contained in the graph of some as-piece of $z$.

We introduce the notation $C^{a s}([a, b), M)$ to denote the set of all as-curves $x:[a, b) \rightarrow M$, where $M$ is a given manifold. In a similar way, we define $C^{a s}((a, b], M), C^{a s}((a, b), M)$, $C^{a s}([a, b], M)$, and also $C^{\text {pas }}([a, b), M), C^{\text {pas }}((a, b], M), C^{\text {pas }}((a, b), M), C^{\text {pas }}([a, b], M)$.

Lemma 4.10 (a) Every pas-curve in the manifold $M$, say $x(t), t \in[a, b),(t \in(a, b]$, $t \in[a, b], t \in(a, b))$, is a subanalytic curve in $M$.

(b) Let $x(t), t \in[a, b)$, be a subanalytic curve in $M$ whose graph is a relatively compact subset of $\mathbb{R} \times M$ and which is left or right continuous at each $t \in[a, b)$. Then there is a, not necessarily unique, pas-decomposition of $x(t)$, that is, a partition of $[a, b]$, say $a=t_{0} \leq t_{1}, \ldots, \leq t_{r}=b$, such that $x \mid\left[t_{i}^{+}, t_{i+1}^{-}\right], i=0, \ldots, r-2$ and $x \mid\left[t_{r-1}^{+}, b\right)$ are as-curves. $A$ similar result holds for subanalytic curves $x(t), t \in(a, b], t \in[a, b]$ or $t \in(a, b)$.

Proof. The proof of $(a)$ is a consequence of the fact that each as-piece of a pas-curve has a semianalytic graph and also the fact that a finite union of semianalytic subsets is a semianalytic subset. To prove $(b)$ we observe first that, because of lemma 4.2, there is a continuous extension, $\bar{x}(t), t \in[a, b]$, of $x(t)$. Also lemma 4.2 implies that for each $t \in[a, b]$ the right and left limits $x\left(t^{-}\right), x\left(t^{+}\right)$exists. Then, by lemma 4.5 for each $t \in(a, b)$ there exists $\epsilon>0$ such that $x\left|\left[t^{+},(t+\epsilon)^{-}\right], x\right|\left[(t+\epsilon)^{+}, t^{-}\right]$are as-curves, and also $x\left|\left[a^{+},(a+\epsilon)^{-}\right], x\right|\left[(b-\epsilon)^{+}, b^{-}\right]$are as-curves for some $\epsilon>0$. Then the proof follows by a standard compactness argument.

Since we want to work within the subanalytic category, it seems that a good choice for a class of possible solutions to a given IDE would be the class of subanalytic curves in $M$ whose graph is a relatively compact subset and which are left or right continuous at each point. The decomposition, predicted in lemma 4.10, of such a curve in as-pieces, which gives its structure as a pas-curve in $M$, which is not unique, is what will enable us to define, in the next paragraph, how such curves can be interpreted as being pas-solutions to a given IDE. In other words, one convenient class of possible solutions to a given IDE would be the class of subanalytic curves having a relatively compact graph, interpreted 
as pas-curves, according to lemma 4.10. As another choice for a class of possible solutions one could choose the class of all pas-curves in $M$. We are going to work also with a bigger class of curves, namely the class of curves that are locally-compact-subanalytic, or lcscurves, in the sense that their restriction to any compact subinterval is a subanalytic curve whose graph is compact and which are left or right continuous at each point. More precisely, we define

Definition 4.11 Let $M$ be a given manifold. Then we define the following classes of locally-compact-subanalytic curves, also called lcs-curves:

(a) $C^{\text {lcs }}\left(\left[t_{0}, t_{1}\right), M\right)$, where $t_{1} \in \mathbb{R}$ or $t_{1}=+\infty$ is the set of all functions $x:\left[t_{0}, t_{1}\right) \rightarrow M$ such that $x \mid\left[t_{0}, t_{2}\right]$ is a pas-curve, for each $t_{2} \in\left(t_{0}, t_{1}\right)$.

(b) $C^{\text {lcs }}\left(\left(t_{0}, t_{1}\right], M\right)$, where $t_{0} \in \mathbb{R}$ or $t_{0}=-\infty$ is the set of all functions $x:\left(t_{0}, t_{1}\right] \rightarrow M$ such that $x \mid\left[t_{2}, t_{1}\right]$ is a pas-curve, for each $t_{2} \in\left(t_{0}, t_{1}\right)$.

(c) $C^{\text {lcs }}\left(\left(t_{0}, t_{1}\right), M\right)$, where $t_{0} \in \mathbb{R}$ or $t_{0}=-\infty$ and $t_{1} \in \mathbb{R}$ or $t_{1}=+\infty$ is the set of all functions $x:\left(t_{0}, t_{1}\right) \rightarrow M$ such that $x \mid\left[\bar{t}_{0}, \bar{t}_{2}\right]$ is a pas-curve, for each $\left[\bar{t}_{0}, \bar{t}_{1}\right] \subseteq\left(t_{0}, t_{1}\right)$.

(d) $C^{\text {lcs }}\left(\left[t_{0}, t_{1}\right], M\right)$, where $t_{0} \in \mathbb{R}$ and $t_{1} \in \mathbb{R}$ is the set of all functions $x:\left[t_{0}, t_{1}\right] \rightarrow M$ such that $x$ is a pas-curve.

We may define the notion of an as-piece and also the notion of a refinement of a given lcs-curve in a similar way as we did in the case of a pas-curve.

Remark. (i) With the notation of definition 4.11, we have, respectively for $(a),(b),(c)$ and $(d)$, that each one of the graphs, graph $\left(x \mid\left[t_{0}, t_{2}\right]\right), \operatorname{graph}\left(x \mid\left[t_{2}, t_{1}\right]\right), \operatorname{graph}\left(x \mid\left[\bar{t}_{0}, \bar{t}_{1}\right]\right)$ and $\operatorname{graph}\left(x \mid\left[t_{0}, t_{1}\right]\right)$ is compact.

(ii) In view of lemma 4.10 we may replace pas-curve by subanalytic curve which is left or right continuous at each point in definition 4.11 and we will obtain an equivalent definition. The decomposition of each subanalytic curve $x\left|\left[t_{0}, t_{2}\right], x\right|\left[t_{2}, t_{1}\right], x \mid\left[\bar{t}_{0}, \bar{t}_{1}\right]$ or $x \mid\left[t_{0}, t_{1}\right]$ as a pas-curve in $M$ is not unique. For a given lcs-curve in $M$ there is a decomposition in at most a countable number of as-pieces, which is not unique.

Solutions to IDE: as-solutions, pas-solutions and lcs-solutions. Now we introduce the notion of solution to a given IDE which is convenient for the purposes of the present paper.

Definition 4.12 (a) An as-solution $x(t), t \in\left[t_{0}, t_{1}\right)$ in $M$ to a given $\operatorname{IDE}(a, f)$ in $M$ is an as-curve in $M$ which satisfies $(a, f)$ for all $t \in\left(t_{0}, t_{1}\right)$, that is, $a(x(t)) \dot{x}(t)=f(x(t))$, for all $t \in\left(t_{0}, t_{1}\right)$. Similar statements hold for as-solutions $x(t)$ in $M$ where $t \in\left(t_{0}, t_{1}\right]$, $t \in\left(t_{0}, t_{1}\right)$ or $t \in\left[t_{0}, t_{1}\right]$ with $t_{0} \neq t_{1}$.

(b) A pas-solution in $M$ to a given IDE is a pas-curve in $M$ such that each open nonempty 
as-piece is an as-solution in $M$.

(c) A lcs-solution in $M$ to a given IDE is a curve in $M$ belonging to $C^{l c s}\left(\left[t_{0}, t_{1}\right), M\right)$, $C^{l c s}\left(\left(t_{0}, t_{1}\right], M\right), C^{l c s}\left(\left(t_{0}, t_{1}\right), M\right)$ or $C^{l c s}\left(\left[t_{0}, t_{1}\right], M\right)$ such that each restriction to a compact subinterval has a pas-decomposition which is a pas-solution in $M$.

Lifted and projected solutions. We have the following result.

Lemma $4.13(a)$ Let $y(t), t \in\left[t_{0}, t_{1}\right),\left(t \in\left(t_{0}, t_{1}\right],\right)$ be a given as-solution in $M^{k}$ to the system $\left(a_{k}, f_{k}\right)$ described in the previous section, for some $k=1,2, \ldots q$. Then for each $t_{2} \in\left(t_{0}, t_{1}\right), y(t)$ is projected via $\pi_{k-1}$ into an as-solution $x(t)$ to the system $\left(a_{k-1}, f_{k-1}\right)$, $x(t)=\pi_{k-1}(y(t)), t \in\left[t_{0}, t_{2}\right]$, (respectively, $\left.t \in\left[t_{2}, t_{1}\right]\right)$, in $M^{k-1}$.

(b) Assume that we have an as-solution $y(t), t \in\left[t_{0}, t_{1}\right),\left(t \in\left(t_{0}, t_{1}\right]\right)$, in $M^{k}$, for some $k=1,2, \ldots q$, to the system $\left(a_{k}, f_{k}\right)$ described in the previous section. Then for each $s=0, \ldots, k-1, y(t)$ is projected via $\pi_{s} \circ \ldots \circ \pi_{k-1}$ into an as-solution $x(t)$ to the system $\left(a_{s}, f_{s}\right), x(t)=\pi_{s} \circ \ldots \circ \pi_{k-1}(y(t)), t \in\left[t_{0}, t_{2}\right]$, (respectively, $\left.t \in\left[t_{2}, t_{1}\right],\right)$, in $M^{s}$, for each $t_{2} \in\left(t_{0}, t_{1}\right)$.

Proof. Part $(a)$ is easy to prove using the fact that $\pi_{k-1}$ is an analytic map, and also lemma 4.6. (c). Part (b) follows using $(a)$.

¿From the previous lemma we can deduce that if $y(t), t \in\left[t_{0}, t_{1}\right)$ is an as-solution to $\left(a_{k}, f_{k}\right)$ in $M^{k}$, for some $k=1, \ldots, q$ and $x(t)=\pi_{s} \circ, \ldots, \pi_{k}(y(t))$ is an as-curve in $M^{s}$, for some $s=1, \ldots, k$, then $x(t), t \in\left[t_{0}, t_{1}\right)$ is an as-solution to $\left(a_{s}, f_{s}\right)$. We will call $y(t)$ a lifted as-solution of $x(t)$, and $x(t)$ the projected as-solution of $y(t)$. A similar definition holds for the case of as-solutions $y(t), t \in\left(t_{0}, t_{1}\right], t \in\left(t_{0}, t_{1}\right), t \in\left[t_{0}, t_{1}\right]$. The notions of a projected and lifted pas-solution are defined as follows. A pas-solution to $\left(a_{k}, f_{k}\right)$, say $y(t) \in M^{k}, t \in\left[t_{0}, t_{1}\right)$ is a lifted pas-solution of a pas-solution $x(t)$ to $\left(a_{s}, f_{s}\right)$ if each as-piece of $y(t)$ is projected, via the composition $\pi_{s} \circ \ldots \circ \pi_{k-1}$, onto an as-piece of $x(t)=\pi_{s} \circ \ldots \circ \pi_{k-1} y(t)$, which is then an as-solution to $\left(a_{s}, f_{s}\right)$. We call $x(t)$ the projected pas-solution of $y(t)$. A similar definition holds for the case of pas-solutions $y(t), t \in\left(t_{0}, t_{1}\right], t \in\left(t_{0}, t_{1}\right), t \in\left[t_{0}, t_{1}\right]$.

\section{Main results.}

Theorem $4.14(a)$ Let $y(t), t \in\left[t_{0}, t_{1}\right)$ (respectively, $t \in\left(t_{0}, t_{1}\right]$,) be an as-solution to $\left(a_{k}, f_{k}\right)$ in $M^{k}, k=1, \ldots, q$. Then $x(t)=\pi_{k-1}(y(t)), t \in\left[t_{0}, t_{2}\right]$ (respectively, $t \in\left[t_{2}, t_{1}\right]$ ) is an as-solution to $\left(a_{k-1}, f_{k-1}\right)$ in $M^{k-1}$, for each $t_{2} \in\left(t_{0}, t_{1}\right)$. The previous statement holds true if we replace an as-solution by a pas-solution or a lcs-solution.

(b) If $x(t), t \in\left[t_{0}, t_{1}\right)$ (respectively, $\left.t \in\left(t_{0}, t_{1}\right]\right)$ is an as-solution to $\left(a_{k-1}, f_{k-1}\right)$ in $M^{k-1}$ such that $x(t) \in M_{0}^{k-1}, t \in\left[t_{0}, t_{1}\right)$ (respectively, $\left.t \in\left(t_{0}, t_{1}\right]\right), k=1, \ldots, q$ then there exists 
$t_{2} \in\left(t_{0}, t_{1}\right)$ and a lifted as-solution $y(t), t \in\left[t_{0}, t_{2}\right]$ (respectively, $t \in\left[t_{2}, t_{1}\right]$ ) of $x \mid\left[t_{0}, t_{2}\right]$ (respectively, $\left.x \mid\left[t_{2}, t_{1}\right]\right)$ to $\left(a_{k}, f_{k}\right)$ in $M^{k}$, in particular, $x(t)=\pi_{k-1}(y(t)), t \in\left[t_{0}, t_{2}\right]$ (respectively, $t \in\left[t_{2}, t_{1}\right]$.)

(c) Let $x(t), t \in\left[t_{0}, t_{1}\right]$ be an as-solution to $\left(a_{k-1}, f_{k-1}\right)$ in $M^{k-1}$ such that $x(t) \in M_{0}^{k-1}$, $t \in\left[t_{0}, t_{1}\right], k=1, \ldots, q$. Then there is a lifted pas-solution of a refinement of $x$, say $y(t), t \in\left[t_{0}, t_{1}\right]$ to $\left(a_{k}, f_{k}\right)$ in $M^{k}$, in particular, $x(t)=\pi_{k-1}(y(t)), t \in\left[t_{0}, t_{1}\right]$. Moreover, if $C \subseteq \pi_{k-1}^{-1}\left(x\left(\left[t_{0}, t_{1}\right]\right)\right), k=1, \ldots, q$, is a compact semianalytic subset such that $\pi_{k-1}(C)=x\left(\left[t_{0}, t_{1}\right]\right)$ then there is a lifted pas-solution of a refinement of $x$, say $y(t) \in C$, $t \in\left[t_{0}, t_{1}\right]$, to $\left(a_{k}, f_{k}\right)$ in $M^{k}$, in particular, $x(t)=\pi_{k-1}(y(t)), t \in\left[t_{0}, t_{1}\right]$.

(d) Let $x(t), t \in\left[t_{0}, t_{1}\right]$ be a pas-solution to $\left(a_{k-1}, f_{k-1}\right)$ in $M^{k-1}$ such that $x(t) \in M_{0}^{k-1}$, $t \in\left[t_{0}, t_{1}\right], k=1, \ldots, q$. Then there is a lifted pas-solution of a refinement of $x$, say $y(t), t \in\left[t_{0}, t_{1}\right]$ to $\left(a_{k}, f_{k}\right)$ in $M^{k}$, in particular, $x(t)=\pi_{k-1}(y(t)), t \in\left[t_{0}, t_{1}\right]$. Moreover, if $C \subseteq \pi_{k-1}^{-1}\left(x\left(\left[t_{0}, t_{1}\right]\right)\right), k=1, \ldots, q$, is a compact semianalytic subset such that $\pi_{k-1}(C)=x\left(\left[t_{0}, t_{1}\right]\right)$, then there is a lifted pas-solution of a refinement of $x$, say $y(t) \in C$, $t \in\left[t_{0}, t_{1}\right]$, to $\left(a_{k}, f_{k}\right)$ in $M^{k}$, in particular, $x(t)=\pi_{k-1}(y(t)), t \in\left[t_{0}, t_{1}\right]$.

(e) Let $x(t), t \in\left[t_{0}, t_{1}\right),\left(t \in\left(t_{0}, t_{1}\right], t \in\left[t_{0}, t_{1}\right], t \in\left(t_{0}, t_{1}\right)\right)$ be a lcs-solution to $\left(a_{k-1}, f_{k-1}\right)$ in $M^{k-1}$, such that $x(t) \in M_{0}^{k-1}, t \in\left[t_{0}, t_{1}\right)$, (respectively, $t \in\left(t_{0}, t_{1}\right], t \in\left[t_{0}, t_{1}\right]$, $\left.t \in\left(t_{0}, t_{1}\right)\right), k=1, \ldots, q$. Then there is a lifted lcs-solution of a refinement of $x$, say $y(t), t \in\left[t_{0}, t_{1}\right)$, (respectively $t \in\left(t_{0}, t_{1}\right], t \in\left[t_{0}, t_{1}\right]$ or $\left.t \in\left(t_{0}, t_{1}\right)\right)$ to $\left(a_{k}, f_{k}\right)$ in $M^{k}$, in particular, $x(t)=\pi_{k-1}(y(t)), t \in\left[t_{0}, t_{1}\right),\left(t \in\left(t_{0}, t_{1}\right], t \in\left[t_{0}, t_{1}\right], t \in\left(t_{0}, t_{1}\right)\right)$. Moreover, if $C \subseteq \pi_{k-1}^{-1}\left(x\left(\left[t_{0}, t_{1}\right)\right)\right)$, (respectively, $C \subseteq \pi_{k-1}^{-1}\left(x\left(\left(t_{0}, t_{1}\right]\right)\right), C \subseteq \pi_{k-1}^{-1}\left(x\left(\left[t_{0}, t_{1}\right]\right)\right)$, $\left.C \subseteq \pi_{k-1}^{-1}\left(x\left(\left(t_{0}, t_{1}\right)\right)\right)\right), k=1, \ldots, q$, is a subset such that $\pi_{k-1}(C)=x\left(\left[t_{0}, t_{1}\right)\right)$, (respectively, $\left.\pi_{k-1}(C)=x\left(\left(t_{0}, t_{1}\right]\right), \pi_{k-1}(C)=x\left(\left[t_{0}, t_{1}\right]\right), \pi_{k-1}(C)=x\left(\left(t_{0}, t_{1}\right)\right)\right)$, and, besides, $C \cap \pi_{k-1}^{-1}\left(x\left(\left[\bar{t}_{0}, \bar{t}_{1}\right]\right)\right)$ is compact subanalytic for each compact subinterval $\left[\bar{t}_{0}, \bar{t}_{1}\right] \subseteq\left[t_{0}, t_{1}\right)$ (respectively, $\left.\left[\bar{t}_{0}, \bar{t}_{1}\right] \subseteq\left(t_{0}, t_{1}\right],\left[\bar{t}_{0}, \bar{t}_{1}\right] \subseteq\left[t_{0}, t_{1}\right],\left[\bar{t}_{0}, \bar{t}_{1}\right] \subseteq\left(t_{0}, t_{1}\right)\right)$, then there is a lifted lcs-solution of a refinement of $x$, say $y(t) \in C, t \in\left[t_{0}, t_{1}\right)$, (respectively $t \in\left(t_{0}, t_{1}\right]$, $\left.t \in\left[t_{0}, t_{1}\right], t \in\left(t_{0}, t_{1}\right)\right)$ to $\left(a_{k}, f_{k}\right)$ in $M^{k}$, in particular, $x(t)=\pi_{k-1}(y(t)), t \in\left[t_{0}, t_{1}\right)$, $\left(t \in\left(t_{0}, t_{1}\right], t \in\left[t_{0}, t_{1}\right], t \in\left(t_{0}, t_{1}\right)\right)$.

Proof. We are going to give a detailed proof of the case $k=1$ only, since the cases $k=2, \ldots, q$ can be proven in an entirely similar way. Part $(a)$ is an easy consequence of lemma 4.13. In order to prove $(b),(c),(d)$ and $(e)$ we are going to prove first several facts, namely, $(i),(i i),(i i i),(i v)$ and $(v)$ below. These facts will be proven under the assumption that $x(t) \in M_{0}, t \in\left[t_{0}, t_{1}\right]$, is an as-solution to $(a, f)$ in $M$, the curve $x(t)$ is simple, that is, $x(a) \neq x(b)$ for all $a, b \in\left[t_{0}, t_{1}\right]$ such that $a \neq b$, and moreover, $x:\left[t_{0}, t_{1}\right] \rightarrow x\left(\left[t_{0}, t_{1}\right]\right)$ is an homeomorphism and also $x\left(\left(t_{0}, t_{1}\right)\right)$ is an analytic submanifold and $x \mid\left(t_{0}, t_{1}\right):\left(t_{0}, t_{1}\right) \rightarrow x\left(\left(t_{0}, t_{1}\right)\right)$ is an analytic diffeomorphism. We can assume without loss of generality (for instance, using Whitney embedding theorem) that $M \subseteq U$ and $M^{1} \subseteq V$ are analytic submanifolds of $U$ and $V$, where $U$ and $V$ are real finite di- 
mensional vector spaces.

(i) The map $\pi_{0}$ can be described as the restriction $p \mid$ (graph $\left.\pi_{0}\right)$ to graph $\pi_{0} \subseteq V \times U$ of the projection onto the second factor $p: V \times U \rightarrow U$. Since $p \mid$ (graph $\left.\pi_{0}\right)$ is a proper analytic map we have that $\left(p \mid\left(\operatorname{graph} \pi_{0}\right)\right)^{-1}\left(x\left(\left[t_{0}, t_{1}\right]\right)\right)$ is a compact semianalytic subset of $V \times U$, therefore, according to lemma 3.4 of [30] it is a finite union of relatively compact connected smooth semianalytic subsets $A$ such that for each $A \operatorname{rank}(p \mid A)$ is constant on $A$. Since $\operatorname{dim}\left(x\left(\left[t_{0}, t_{1}\right]\right)=1\right.$, it is easy to see that for each $A \operatorname{rank}(p \mid A)$ is 0 or 1 , and moreover, $x\left(\left[t_{0}, t_{1}\right]\right)$ is the union of those $p(\bar{A})$ such that $p \mid A$ has rank 1 and therefore $p(\bar{A})$ is not a point. We observe that if there is a compact semianaliytic subset $C \subseteq \pi_{0}^{-1}\left(x\left(\left[t_{0}, t_{1}\right]\right)\right)$ such that $\pi_{0}(C)=x\left(\left[t_{0}, t_{1}\right]\right)$ then we have that $C_{1}=\left(p \mid \text { graph } \pi_{0}\right)^{-1}\left(x\left(\left[t_{0}, t_{1}\right]\right)\right) \cap(C \times U)$ is compact and semianalytic. According to lemma 3.4 of [30, $C_{1}$ is a finite union of relatively compact connected smooth semianalytic subsets $A$ having the same properties as before.

(ii) Let $\alpha \in\left[t_{0}, t_{1}\right]$ be fixed. Then there is an $A$, say $A=A_{0}$, such that $x(\alpha) \in p\left(\bar{A}_{0}\right)$. Since $\bar{A}_{0}$ is connected and compact and the curve $x(t), t \in\left[t_{0}, t_{1}\right]$, is simple, we have that $p\left(\bar{A}_{0}\right)$ is homeomorphic to a closed interval (possibly of zero length) via the curve $x$, say $p\left(\bar{A}_{0}\right)=x\left(\left[a_{1}, a_{2}\right]\right)$, where $\left[a_{1}, a_{2}\right] \subseteq\left[t_{0}, t_{1}\right]$. We can assume without loss of generality that $p\left(\bar{A}_{0}\right)$ is homeomorphic to a closed interval of nonzero length.

(iii) By using theorem 6.10 of [30] we can see that for given points $q_{i} \in p^{-1}\left(x\left(a_{i}\right)\right) \cap \bar{A}_{0}$, $i=1,2$, so in particular, we have $q_{1} \neq q_{2}$, there is a continuous semianalytic curve $w(s)$, $s \in\left[s_{0}, s_{1}\right]$, in $\bar{A}_{0}$ such that $w\left(s_{i}\right)=q_{i+1}, i=0,1$. Then $w\left(\left[s_{0}, s_{1}\right]\right)$ is a compact semianalytic subset of $V \times U$ of dimension 1 and we have $\left.p\left(w\left(\left[s_{0}, s_{1}\right]\right)\right)=x\left(\left[a_{1}, a_{2}\right]\right)\right]$. Using lemma 3.4 of [30] we see that since $w\left(\left[s_{0}, s_{1}\right]\right)$ has dimension 1 it is a finite union of relatively compact connected smooth semianalytic subsets $B$ of dimension less or equal than 1 , such that the restriction of the projection $p \mid B$ has constant rank of value 0 or 1 . Since each $\bar{B}$ is connected and compact we have that $p(\bar{B})$ is homeomorphic to a closed interval, say $p(\bar{B})=x\left(\left[a_{B}, b_{B}\right]\right)$. For at least some $B$ such that $p(\bar{B})$ is not a point one must have that $p(\bar{B})$ contains the point $x(\alpha)$. Observe that $p(B)=x\left(\left(a_{B}, b_{B}\right)\right)$ is an analytic submanifold which is a subanalytic subset and that $p \mid B: B \rightarrow x\left(\left(a_{B}, b_{B}\right)\right)$ is an analytic diffeomorphism. This gives, in particular, a parametrization of the analytic submanifold $B$ with the parameter $t$, namely, $z(t)=(p \mid B)^{-1}(x(t)), t \in\left(a_{B}, b_{B}\right)$. We can give a definition of graph $\left((p \mid B)^{-1} \circ x\right)$ as a subanalytic subset of $\mathbb{R} \times M^{1} \times M$ as follows. We have graph $\left((p \mid B)^{-1} \circ x\right)=\left\{(t, z) \in \mathbb{R} \times M^{1} \times M: z \in B, p(z)=x,(t, x) \in\right.$ $\left.\operatorname{graph}\left(x \mid\left(a_{B}, b_{B}\right)\right)\right\}$, which defines graph $\left((p \mid B)^{-1} \circ x\right)$ by subanalytic conditions, since $B$ is a semianalytic subset of $M^{1} \times M$, graph $x$ is a subanalytic subset of $\mathbb{R} \times M$, by def- 
inition, and $\operatorname{graph}\left(x \mid\left(a_{B}, b_{B}\right)\right)=\left\{(t, x) \in \mathbb{R} \times M:(t, x) \in \operatorname{graph} x, t \in\left(a_{B}, b_{B}\right)\right\}$. Then using lemma 4.2 we can deduce that there is a uniquely defined continuous extension, which we will call $z$ by a slight abuse of notation, $z(t), t \in\left[a_{B}, b_{B}\right]$, whose image is the semianalytic subset $\bar{B}=z\left(\left[a_{B}, b_{B}\right]\right)$, which is an as-curve in $M^{1} \times M$. We have, in particular, that $z(t), t \in\left[a_{B}, b_{B}\right]$ is an as-curve such that $p(z(t))=x(t), t \in\left[a_{B}, b_{B}\right]$. It is clear that the extension $z(t), t \in\left[a_{B}, b_{B}\right]$ is given by $(p \mid \bar{B})^{-1} \circ x$

(iv) Assume that $x(\alpha) \in p(B)$. We have the as-curve $z(t)=(y(t), x(t)), t \in\left[a_{B}, b_{B}\right]$ in $\operatorname{graph}\left(\pi_{0}\right)$, therefore $p(z(t))=x(t)$, for $t \in\left[a_{B}, b_{B}\right]$, then the curve $y(t)$ satisfies $\pi_{0}(y(t))=x(t), t \in\left[a_{B}, b_{B}\right]$. Using this it becomes clear from the definition of $\left(a_{1}, f_{1}\right)$ that $y(t)$ satisfies the system $\left(a_{1}, f_{1}\right)$, for $t \in\left(a_{B}, b_{B}\right)$. We have that, for any $\left[\alpha-\epsilon_{1}, \alpha+\epsilon_{2}\right] \subseteq\left[a_{B}, b_{B}\right]$, where $\epsilon_{1}, \epsilon_{2} \geq 0, y(t), t \in\left[\alpha-\epsilon_{1}, \alpha+\epsilon_{2}\right]$ is an as-curve in $M^{1}$. In fact, this is a direct consequence of lemma 4.6. (c), since the projection $q: M^{1} \times M \rightarrow M^{1}$ is an analytic map. It is clear that $y(t)$ satisfies the system $\left(a_{1}, f_{1}\right)$, for $t \in\left(\alpha-\epsilon_{1}, \alpha+\epsilon_{2}\right)$.

(v) Assume now that $x(\alpha) \in p(\bar{B})-p(B)$, then $\alpha=a_{B}$ or $\alpha=b_{B}$. If $\alpha=a_{B}$ (respectively $\alpha=b_{B}$ ) we can proceed in a similar way as we did in $(i v)$ and we have an as-curve $z(t)=(y(t), x(t)), t \in\left[\alpha, \alpha+\epsilon_{2}\right]$ (respectively, $t \in\left[\alpha-\epsilon_{1}, \alpha\right]$ ) in $\operatorname{graph}\left(\pi_{0}\right)$, then, in particular, $p(z(t))=x(t), t \in\left[\alpha, \alpha+\epsilon_{2}\right]$, (respectively, $\left.t \in\left[\alpha-\epsilon_{1}, \alpha\right)\right]$. Then the curve $y(t)=q(z(t))$ satisfies $\pi_{0}(y(t))=x(t), t \in\left[\alpha, \alpha+\epsilon_{2}\right]$ (respectively, $t \in\left[\alpha-\epsilon_{1}, \alpha\right]$ ) and is an as-curve. It is clear that $y(t)$ satisfies the system $\left(a_{1}, f_{1}\right)$, for $t \in\left(\alpha, \alpha+\epsilon_{2}\right)$ (respectively $t \in\left(\alpha-\epsilon_{1}, \alpha\right)$ ).

We are going to prove $(b)$. If $x(t)=x\left(t_{0}\right)$ is a constant then it can be lifted to a constant curve $y(t)=y\left(t_{0}\right)$, where $y\left(t_{0}\right) \in \pi_{0}^{-1}\left(x\left(t_{0}\right)\right)$, which solves the problem, so let us assume that $x(t)$ is not a constant. By conveniently lowering the value of $t_{1}$ we can assume without loss of generality that $x(t) \in M_{0}, t \in\left[t_{0}, t_{1}\right]$, is an as-solution to $(a, f)$ in $M$. Moreover, by lowering the value of $t_{1}$ if necessary and using lemma 4.7 we can assume that the curve $x(t)$ is simple, that is $x(a) \neq x(b)$ for all $a, b \in\left[t_{0}, t_{1}\right]$ such that $a \neq b$, and moreover, that $x:\left[t_{0}, t_{1}\right] \rightarrow x\left(\left[t_{0}, t_{1}\right]\right)$ is an homeomorphism onto $x\left(\left[t_{0}, t_{1}\right]\right), x\left(\left(t_{0}, t_{1}\right)\right)$ is an analytic submanifold of $M$ and $x \mid\left(t_{0}, t_{1}\right):\left(t_{0}, t_{1}\right) \rightarrow x\left(\left(t_{0}, t_{1}\right)\right)$ is an analytic diffeomorphism. By using $(v)$ with $\alpha=t_{0}$, we must have $a_{B}=t_{0}$ and then the proof of $(b)$ follows from $(v)$ by taking $\alpha+\epsilon_{2}=t_{2}$. The case of an interval $\left(t_{0}, t_{1}\right]$ can be proved in an entirely similar way.

We are going to prove $(c)$. As in the proof of $(b)$, the case in which $x$ is constant is trivial, so we shall assume that $x$ is not a constant. Using $(b)$ we can conclude that for each $\bar{t} \in\left(t_{0}, t_{1}\right)$ there are intervals $\left[\bar{t}-\epsilon_{1}, \bar{t}\right] \subseteq\left[t_{0}, t_{1}\right]$ and $\left[\bar{t}, \bar{t}+\epsilon_{2}\right] \subseteq\left[t_{0}, t_{1}\right]$, where 
$\epsilon_{1}, \epsilon_{2}>0$, and lifted as-solutions $y_{\bar{t}}^{-}(t), t \in\left[\bar{t}-\epsilon_{1}, \bar{t}\right]$, and $y_{\bar{t}}^{+}(t), t \in\left[\bar{t}, \bar{t}+\epsilon_{2}\right]$, in $M^{1}$, of $x \mid\left[\bar{t}-\epsilon_{1}, \bar{t}\right]$ and $x \mid\left[\bar{t}, \bar{t}+\epsilon_{2}\right]$ to $\left(a_{1}, f_{1}\right)$. Also if $\bar{t}=t_{0}\left(\bar{t}=t_{1}\right)$ there is a lifted assolution $y_{t_{0}}^{+}(t)$ of $x \mid\left[t_{0}, t_{0}+\epsilon_{2}\right]$ (respectively, $y_{t_{1}}^{-}(t)$ of $\left.x \mid\left[t_{1}-\epsilon_{1}, t_{1}\right],\right)$ to $\left(a_{1}, f_{1}\right)$. Using this and a compactness argument we can conclude that there is a partition of $\left[t_{0}, t_{1}\right]$, say, $t_{0}=\bar{t}_{0}<\bar{t}_{1}, \ldots,<\bar{t}_{r}=t_{1}$, such that there are lifted as-solutions $y_{i}(t), t \in\left[\bar{t}_{i}, \bar{t}_{i+1}\right]$, of $x \mid\left[\bar{t}_{i}, \bar{t}_{i+1}\right]$, to $\left(a_{1}, f_{1}\right), i=0, \ldots, r-1$. Then a lifted pas-solution can be obtained by gluing together the lifted solutions $y_{i}(t), i=0, \ldots, r-1$ into a single pas-solution $y(t), t \in\left[t_{0}, t_{1}\right]$, which is always possible, and in more than one way if $r>0$. If there is a set $C$ satisfying the conditions stated in $(c)$ then we can prove that there is a lifted pas-solution satisfying the required conditions proceeding as above and taking into account the comments made at the end of $(i)$.

The proof of $(d)$ can be obtained as a consequence of $(c)$ by conveniently gluing together a finite number of lifted pas-solutions each one corresponding to each as-piece of the given pas-solution.

The proof of $(e)$ can be obtained as a consequence of $(d)$ by conveniently gluing together conveniently chosen at most countable lifted pas-solutions.

The next theorem adds some more information and completes the picture of the relationship between solutions and lifted solutions to a given IDE.

Let $x(t), t \in[\alpha, \beta]$ be an as-solution to $(a, f)$ in $M^{k}$, for some $k=0, \ldots, q-1$. If for some $s_{0} \in[\alpha, \beta]$ we have $x\left(s_{0}\right) \in M_{2}^{k}$ then we must have that there is at most a finite number of $t \in[\alpha, \beta]$, say $\alpha \leq t_{1}<\ldots<t_{r} \leq \beta$, such that $x\left(t_{i}\right) \in M_{0}^{k}$. This is because $M_{0}^{k}$ is defined by analytic equations. In this case we will call $x$ a normal as-solution to $\left(a_{k}, f_{k}\right)$ in $M^{k}$. If $x(t), t \in[\alpha, \beta]$ is a pas-solution to $\left(a_{k}, f_{k}\right)$ in $M^{k}$, for some $k=0, \ldots, q-1$ such that all its as-pieces are normal we will call $x$ a normal pas-solution to $\left(a_{k}, f_{k}\right)$ in $M^{k}$.

Let us introduce the following notation. $\tilde{M}=\bigsqcup_{k=0}^{q} M^{k}$, where $\bigsqcup$ means disjoint union, $\tilde{\pi}: \tilde{M} \rightarrow M$, where $\tilde{\pi}(x)=\pi_{k}(x)$ if $x \in M^{k}$. Now assume that we have a finite collection of normal pas-solutions of the following type. For each $i=1, \ldots, r$ let $y_{i}(t), t \in\left[t_{i, 0}, t_{i, 1}\right]$ be a normal pas-solution to some of the IDE $\left(a_{k}, f_{k}\right), k=0, \ldots, q$, say $\left(a_{k_{i}}, f_{k_{i}}\right)$, where $k_{i} \in\{0, \ldots, q\}, i=1, \ldots, r$, so that $y_{i}(t) \in M_{0}^{k_{i}}$ only for at most a finite number of $t \in\left[t_{i, 0}, t_{i, 1}\right]$. Assume, without loss of generality, that $t_{i, 1}=t_{i+1,0}$, for $i=1, \ldots, r-1$, so we have a partition $t_{1,0}<t_{1,1}=t_{2,0}<\ldots<t_{r, 1}$ of the interval $\left[t_{1,0}, t_{r, 1}\right]$. Then the collection of curves $y_{i}, i=1, . ., r$ may be thought of as a single pas-curve in $\tilde{M}$, say $y(t)$, $t \in\left[t_{1,0}, t_{r, 1}\right]$. We will say that $y(t), t \in\left[t_{1,0}, t_{r, 1}\right]$ is a normal pas-solution to $(\tilde{a}, \tilde{f})$ in $\tilde{M}$. It follows from theorem 4.14 $(a)$ that $x(t), t \in\left[t_{1,0}, t_{r, 1}\right]$, given by $x(t)=\pi_{0} \circ \ldots \circ \pi_{k_{i}-1} y_{i}(t)$, 
$t \in\left[t_{i, 0}, t_{i, 1}\right], i=1, . ., r$, or, equivalently, $x(t)=\tilde{\pi}(y(t)), t \in\left[t_{1,0}, t_{r, 1}\right]$, is a pas-solution to $(a, f)$. In this situation we will say that the solution $y$ in $\tilde{M}$ is a normal pas-lifting of the solution $x$ in $M$, or, equivalently, that $y$ is a lifted pas-solution of $x$, and also that $x$ is the pas-projection of $y$.

We can use the previous remarks in combination with theorem 4.14 and we obtain the following theorem

Theorem 4.15 Let $x(t), t \in[\alpha, \beta]$ be an as-solution to $(a, f)$. Then there is a lifted normal pas-solution $y(t), t \in[\alpha, \beta]$ to $(\tilde{a}, \tilde{f})$ in $\tilde{M}$ of a refinement of $x(t)$.

Equivalence between an $\operatorname{IDE}(a, f)$ in $M$ and $\left(\tilde{a}_{2}, \tilde{f}_{2}\right)$ in $\tilde{M}_{2}$. Using the notation introduced in section 3 we have obviously that $\tilde{M}_{2}$ is an open subset of $\tilde{M}, \tilde{\pi}_{2}=\tilde{\pi} \mid \tilde{M}_{2}$, $\left(\tilde{a}_{2}, \tilde{f}_{2}\right)=(\tilde{a}, \tilde{f}) \mid \tilde{M}_{2}$.

Let $y(t), t \in[\alpha, \beta]$, be a normal as-solution to $\left(a_{k}, f_{k}\right)$ in $M^{k}$ and let $\alpha \leq t_{1}<\ldots<$ $t_{r} \leq \beta$, be the set of all $t \in[\alpha, \beta]$ such that $x\left(t_{i}\right) \in M_{0}^{k}$, as explained before the theorem. Then we obtain a well defined collection of as-solutions to $\left(a_{k}, f_{k}\right) \mid M_{2}^{k}$ in $M_{2}^{k}$, namely, $y\left|\left(t_{i}, t_{i+1}\right), i=1, \ldots, r-1, y\right|\left(\alpha, t_{1}\right)$, if $\alpha<t_{1}, y \mid\left(t_{r}, \beta\right)$, if $t_{r}<\beta$. We will call this collection of solutions the collection of solutions in $M_{2}^{k}$, or, also, in $\tilde{M}_{2}$, induced by the as-solution $y$. It is clear that by continuous extension, using lemma 4.2, of each $y\left|\left(t_{i}, t_{i+1}\right), i=1, \ldots, r-1, y\right|\left(\alpha, t_{1}\right)$, if $\alpha<t_{1}, y \mid\left(t_{r}, \beta\right)$, if $t_{r}<\beta$, we obtain a refinement of $x$, with as-piece closures $y\left|\left[t_{i}^{+}, t_{i+1}^{-}\right], i=1, \ldots, r-1, y\right|\left[\alpha^{+}, t_{1}^{-}\right]$, if $\alpha<t_{1}, y \mid\left[t_{r}^{+}, \beta^{-}\right]$, if $t_{r}<\beta$. Of course, by continuity of $y$ we have $y\left|\left[t_{i}^{+}, t_{i+1}^{-}\right]=y\right|\left[t_{i}, t_{i+1}\right], i=1, \ldots, r-1$, $y\left|\left[\alpha^{+}, t_{1}^{-}\right]=y\right|\left[\alpha, t_{1}\right]$, if $\alpha<t_{1}, y\left|\left[t_{r}^{+}, \beta^{-}\right]=y\right|\left[t_{r}, \beta\right]$, if $t_{r}<\beta$.

Given any normal pas-solution $y$ to $\left(a_{k}, f_{k}\right)$ in $M^{k}$, we shall define the collection of assolutions in $M_{2}^{k}$, or, also, in $\tilde{M}_{2}$, induced by the normal pas-solution $y$ as being the union of the collections of as-solutions induced by all the as-pieces of $y$. Finally, given any normal pas-solution $y$ to $(\tilde{a}, \tilde{f})$, in $\tilde{M}$. we shall define the collection of as-solutions in $\tilde{M}_{2}$ induced by the normal pas-solution $y$ as being the union of the collections of as-solutions induced by all the as-pieces of which $y$ is ultimately composed.

It is clear that a given normal pas-solution $y(t), t \in[\alpha, \beta]$ to $(\tilde{a}, \tilde{f})$ in $\tilde{M}$, say $y(t) \in M^{k}$, for some $k=0, \ldots, q$ as before, cannot be completely recovered from its induced collection of as-solutions to $\left(\tilde{a}_{2}, \tilde{f}_{2}\right)$ in $\tilde{M}_{2}$. This is because even if it is known that the value of $y(t)$ at some $t \in[\alpha, \beta]$ is the left or the right limit at $t$ of some of the as-pieces of the induced collection of as-curves by $y$, it is not known which one of those limits, unless one has some additional information, like continuity of $y$ at $t$. Modulo this loss of information, 
we can consider the IDE $\left(\tilde{a}_{2}, \tilde{f}_{2}\right)$ with domain $\tilde{M}_{2}$ and range $F$ and $(\tilde{a}, \tilde{f})$ with domain $\tilde{M}$ and range $F$ as being equivalent. In this sense, the meaning of theorems 4.14 and 4.15 is that solving a given analytic IDE $(a, f)$ with domain $M$ and range $F$ can be reduced essentially to solving a finite collection of IDE of constant rank, namely, $\left(a_{i}, f_{i}\right) \mid M_{2}^{i}$, $i=0, \ldots, q-1$, or, equivalently, the system of constant $\operatorname{rank}\left(\tilde{a}_{2}, \tilde{f}_{2}\right)$ with domain $\tilde{M}_{2}$ and range $F$. As we have said before it is clear that each $\left(a_{i}, f_{i}\right) \mid M_{2}^{i}, i=0, \ldots, q-1$ defines an analytic family of vector fields and hence an analytic affine distribution of constant rank on the manifold $M_{2}^{i}$. This immediately implies that $\left(\tilde{a}_{2}, \tilde{f}_{2}\right)$ defines an analytic family of vector fields and hence an analytic affine distribution of constant rank on the manifold $\tilde{M}_{2}$. Therefore, it also gives rise to an analytic control system, [31]. The usefulness of this kind of result relies obviously on the interest that such control systems have in several fields. For instance, the well known theorem of Sussmann, 42, tells us how to deal with problems of reachability and observability, once one has a control system. See also 31] where this kind of questions are solved in the context of subanalytic sets.

Reparametrization and extension of solutions. First we shall define the notion of a reparametrization in the context of as-curves. A reparametrization of an as-curve $x(t), t \in\left[t_{0}, t_{1}\right)$, in $M$, is a change of variables $t=\tau(s), s \in\left[s_{0}, s_{1}\right),\left(s \in\left(s_{1}, s_{0}\right]\right)$ such that $\tau:\left[s_{0}, s_{1}\right) \rightarrow \mathbb{R}$ (respectively, $\tau:\left(s_{1}, s_{0}\right] \rightarrow \mathbb{R}$ ) is an as-curve in $\mathbb{R}$ which is also an homeomorphism onto $\left[t_{0}, t_{1}\right)$ such that $\tau\left(s_{0}\right)=t_{0}$ and $\tau \mid\left(s_{0}, s_{1}\right):\left(s_{0}, s_{1}\right) \rightarrow\left(t_{0}, t_{1}\right)$ (respectively, $\left.\tau:\left(s_{1}, s_{0}\right) \rightarrow\left(t_{0}, t_{1}\right)\right)$ is an analytic diffomorphism. It is easy to prove that in this case the composition $(x \circ \tau)(s), s \in\left[s_{0}, s_{1}\right)$ (respectively, $s \in\left(s_{1}, s_{0}\right]$ ) is an as-curve in $M$. Similar definitions and results hold for the case of as-curves $x(t)$ whose domain is an interval of the type $\left(t_{0}, t_{1}\right],\left(t_{0}, t_{1}\right),\left[t_{0}, t_{1}\right]$.

Let $(a, f)$ be a given IDE with domain $M$ and range $F$. By definition, $(a, f)$ is homogeneous if $f=0$. It is clear that if $x(t), t \in\left[t_{0}, t_{1}\right)$ is a given as-solution to an homogeneous system $(a, 0)$ and $t=\tau(s), s \in\left[s_{0}, s_{1}\right)\left(s \in\left(s_{1}, s_{0}\right]\right)$ is a given reparametrization then the curve $y(s) \equiv x \circ \tau(s), s \in\left[s_{0}, s_{1}\right)$ (respectively, $s \in\left(s_{1}, s_{0}\right]$ ) is also an as-solution to $(a, 0)$. More generally, if $(a, f)$ is not necessarily homogeneous then $y(s)$ satisfies $a(y(s)) \dot{y}(s)=(d \tau / d s) f(y(s)), s \in\left(s_{0}, s_{1}\right)$, or, using a different and also standard notation, $a(y(s)) \dot{y}(s)=\dot{t}(s) f(y(s)), s \in\left(s_{0}, s_{1}\right)$. Similar results hold for the case of as-curves whose domain is an interval of the type $\left(t_{0}, t_{1}\right],\left(t_{0}, t_{1}\right)$, or $\left[t_{0}, t_{1}\right]$.

Theorem 4.16 (a) Let $(a, 0)$ be a given homogeneous IDE with domain $M$ and range $F$. Let $x_{+}(t), t \in\left[t_{0}, t_{1}\right)$, be an as-solution to $(a, 0)$ in $M$, which is not a constant. Then there is an as-solution $z(s), s \in\left(s_{0}-\delta_{1}, s_{0}+\delta_{2}\right)$, in $M$, for some $\delta_{1}, \delta_{2}>0$, satisfying all the conditions stated in lemma 4.9. More precisely, $z\left(s_{0}\right)=x_{+}\left(t_{0}\right), z\left(\left[s_{0}, s_{0}+\delta_{2}\right)\right)=x_{+}\left(\left[t_{0}, t_{2+}\right)\right)$ for some $t_{2+} \in\left(0, t_{1}\right)$ and we also have that $t_{2+}$ and $s_{0}+\delta_{2}$ are such that $x_{+} \mid\left[t_{0}, t_{2+}\right)$ 
and $z \mid\left[s_{0}, s_{0}+\delta_{2}\right)$ are homeomorphisms onto $z\left(\left[s_{0}, s_{0}+\delta_{2}\right)\right)=x_{+}\left(\left[t_{0}, t_{2+}\right)\right), x_{+} \mid\left(t_{0}, t_{2+}\right)$ and $z \mid\left(s_{0}, s_{0}+\delta_{2}\right)$ are analytic diffeomorphisms onto $z\left(\left(s_{0}, s_{0}+\delta_{2}\right)\right)=x_{+}\left(\left(t_{0}, t_{2+}\right)\right)$ which is an analytic submanifold which is also a semianalytic subset and, moreover, the curve in $\mathbb{R}$, say $t=t(s)$, given by $\left(x_{+} \mid\left(t_{0}, t_{2+}\right)\right)^{-1} \circ\left(z \mid\left(s_{0}, s_{0}+\delta_{2}\right)\right):\left(s_{0}, s_{0}+\delta_{2}\right) \rightarrow \mathbb{R}$ is an as-curve in $\mathbb{R}$ which is an analytic diffeomorfism onto its image $\left(t_{0}, t_{2+}\right)$. Moreover, $t_{2+}$ can be chosen such that for each $t_{3+} \in\left(t_{0}, t_{2+}\right], x_{+}\left(\left(t_{0}, t_{3+}\right)\right)$ is a neighborhood of $x_{+}\left(t_{0}\right)$ in $x_{+}\left(\left[t_{0}, t_{2+}\right)\right)-\left\{x_{+}\left(t_{0}\right)\right\}$. Similar results hold for as-solutions $x_{-}(t), t \in\left(t_{0}, t_{1}\right]$.

(b) Let $(a, f)$ be a given IDE with domain $M$ and range $F$. Let $x_{+}(t), t \in\left[t_{0}, t_{1}\right)$, be an as-solution to $(a, f)$ in $M$ which is not a constant. Then there is an as-curve $z(s)$, $s \in\left(s_{0}-\delta_{1}, s_{0}+\delta_{2}\right)$, in $M$, for some $\delta_{1}, \delta_{2}>0$, not necessarily a solution, satisfying all the conditions stated in lemma 4.9, as we have explained in (a), and, besides, the as-curve $z(s), s \in\left[s_{0},, s_{0}+\delta_{2}\right)$ satisfies the equation a $(z(s)) \dot{z}(s)=\dot{t}(s) f(z(s)), s \in\left(s_{0}, s_{0}+\delta_{2}\right)$, with $\dot{t}(s)>0, s \in\left(s_{0}, s_{1}\right)$. Similar results hold for as-solutions of the type $x_{-}(t)$, $t \in\left(t_{0}, t_{1}\right]$.

(c) Let $(a, f)$ be a given IDE with domain $M$ and range $F$. Let $x_{+}(t), t \in\left[t_{0}, t_{1}\right)$, be an as-solution to $(a, f)$ in $M$ which is not a constant and let $z(s)$ be as in $(b)$. Then by conveniently diminishing the value of $\delta_{1}$ and the value of $\delta_{2}$ if necessary, we have the following. There is an as-solution $x_{-}(t), t \in\left(t_{2_{-}}, t_{0}\right]$ to some of the systems $(a, \pm f)$ in $M$ such that $x_{-}\left(t_{0}\right)=x_{+}\left(t_{0}\right)=z\left(s_{0}\right)$, and $x_{-} \mid\left(t_{2_{-}}, t_{0}\right]$ and $z \mid\left(s_{0}-\delta_{1}, s_{0}\right]$ are homeomorphisms onto $z\left(\left(s_{0}-\delta_{1}, s_{0}\right]\right)=x_{-}\left(\left(t_{2-}, t_{0}\right]\right), x_{-} \mid\left(t_{2-}, t_{0}\right)$ and $z \mid\left(s_{0}-\delta_{1}, s_{0}\right)$ are analytic diffeomorphisms onto $z\left(\left(s_{0}-\delta_{1}, s_{0}\right)\right)=x_{-}\left(\left(t_{2-}, t_{0}\right)\right)$, which is an analytic submanifold which is also a semianalytic subset. Moreover, $\left(x_{-} \mid\left(t_{2-}, t_{0}\right)\right)^{-1} \circ\left(z \mid\left(s_{0}-\delta_{1}, s_{0}\right)\right):\left(s_{0}-\delta_{1}, s_{0}\right) \rightarrow \mathbb{R}$ is an as-curve in $\mathbb{R}$ which is an as-diffeomorfism onto its image $\left(t_{2-}, t_{0}\right)$. We also have that $t_{2-}$ can be chosen such that for each $t_{3-} \in\left(t_{2-}, t_{0}\right], x_{-}\left(\left(t_{3-}, t_{0}\right)\right)$ is a neighborhood of $x_{-}\left(t_{0}\right)$ in $x_{-}\left(\left(t_{2-}, t_{0}\right]\right)-\left\{x_{-}\left(t_{0}\right)\right\}$. The as-curve $z(s), s \in\left[s_{0}, s_{0}+\delta_{2}\right)$ satisfies the equation $a(z(s)) \dot{z}(s)=\dot{t}(s) f(z(s)), s \in\left(s_{0}, s_{0}+\delta_{2}\right)$ where $\dot{t}(s)>0, s \in\left(s_{0}, s_{0}+\delta_{2}\right)$. The as-curve $z(s), s \in\left(s_{0}-\delta_{1}, s_{0}\right]$ satisfies the equation a $(z(s)) \dot{z}(s)=\dot{t}(s) f(z(s)), s \in\left(s_{0}-\delta_{1}, s_{0}\right)$ where $\dot{t}(s)>0$ if $x_{-}(t)$ satisfies $(a, f)$ and $\dot{t}(s)<0$ if $x_{-}(t)$ satisfies $(a,-f)$. Similar results hold for as-solutions $x_{-}(t), t \in\left(t_{0}, t_{1}\right]$.

Proof. Part $(a)$ and part $(b)$ are a direct consequence of lemma 4.9. To prove part (c) consider the homogeneous IDE $(\alpha, 0)$ with domain $\mathbb{R} \times M$ and range $F$ where $\alpha(t, x)(\dot{t}, \dot{x})=a(x) \dot{x}-\dot{t} f(x)$. Consider the solution $(t(s), z(s)), s \in\left(s_{0}, s_{0}+\delta_{2}\right)$ to the homogeneous system $(\alpha, 0)$ where $z(s), s \in\left(s_{0}-\delta_{1}, s_{0}+\delta_{2}\right)$ is the as-curve considered in $(b)$. Since we know from $(b)$ that $a(z(s)) \dot{z}(s)$ and $f(z(s)))$ are linearly dependent for $s \in\left(s_{0}, s_{0}+\delta_{2}\right)$ we can conclude, using the analyticity of $z(s), s \in\left(s_{0}-\delta_{1}, s_{0}+\delta_{2}\right)$, that they must also be linearly dependent for $s \in\left(s_{0}-\delta_{1}, s_{0}+\delta_{2}\right)$. We can also show, by using lemma 4.8, that there exist $\delta_{1}$ such that $z\left(\left(s_{0}-\delta_{1}, s_{0}\right]\right)-\left\{z\left(s_{0}\right)\right\}$ is nonempty and locally connected at $z\left(s_{0}\right)$, more precisely, $z\left(\left(s_{0}-\delta_{3}, s_{0}\right)\right)$ is a neigh- 
borhood of $z\left(s_{0}\right)$ in $z\left(\left(s_{0}-\delta_{1}, s_{0}\right]\right)-\left\{z\left(s_{0}\right)\right\}$ for all $s_{0}-\delta_{3} \in\left(s_{0}-\delta_{1}, s_{0}\right)$. Moreover, $\delta_{1}$ can be chosen such that $z \mid\left[s_{0}-\delta_{1}, s_{0}\right]:\left[s_{0}-\delta_{1}, s_{0}\right] \rightarrow z\left(\left[s_{0}-\delta_{1}, s_{0}\right]\right)$ is an homeomorphism, $z\left(\left(s_{0}-\delta_{1}, s_{0}\right)\right)$ is an analytic submanifold which is a semianalytic subset of $M$ and $z:\left(s_{0}-\delta_{1}, s_{0}\right) \rightarrow z\left(\left(s_{0}-\delta_{1}, s_{0}\right)\right)$ is an analytic diffeomorphism. Since we have a linear dependence between $a(z(s)) \dot{z}(s)$ and $f(z(s)), s \in\left(s_{0}-\delta_{1}, s_{0}+\delta_{2}\right)$ we have several cases. Assume first that $a(z(s)) \dot{z}(s)=0, s \in\left(s_{0}, s_{0}+\delta_{2}\right)$. Then since $\dot{t}(s) \neq 0, s \in\left(s_{0}, s_{0}+\delta_{2}\right)$ we can conclude that $f(z(s))=0, s \in\left(s_{0}, s_{0}+\delta_{2}\right)$. By analyticity of $z(s), s \in\left(s_{0}-\delta_{1}, s_{0}+\delta_{2}\right)$ we obtain that $a(z(s)) \dot{z}(s)=0$ and $f(z(s))=0, s \in\left(s_{0}-\delta_{1}, s_{0}+\delta_{2}\right)$. Then to prove $(c)$ in this case we can simply take $t=s-s_{0}+t_{0}, t_{2-}=-\delta_{1}+t_{0}$ and $x_{2-}(t)=z\left(t+s_{0}-t_{0}\right)$. We can proceed in a similar way if we assume that $f(z(s))=0, s \in\left(s_{0}, s_{0}+\delta_{2}\right)$. Let us consider now the case where $a(z(s)) \dot{z}(s), s \in\left(s_{0}, s_{0}+\delta_{2}\right)$ is not identically 0 . We are going to show that, after conveniently diminishing the value of $\delta_{1}$ if necessary, there exists a unique as-curve in $\mathbb{R}, \lambda:\left(s_{0}-\delta_{1}, s_{0}\right) \rightarrow \mathbb{R}$ such that $a(z(s)) \dot{z}(s)=\lambda(s) f(z(s)), s \in\left(s_{0}-\delta_{1}, s_{0}\right)$. First one should take into account that $z(s), s \in\left(s_{0}-\delta_{1}, s_{0}+\delta_{2}\right)$ is analytic then so are $f(z(s))$ and $a(z(s)) \dot{z}(s), s \in\left(s_{0}-\delta_{1}, s_{0}+\delta_{2}\right)$ and therefore they have at most a finite number of isolated zeros in a neighborhood of $s_{0}$. It is easy to see from the equation $a(z(s)) \dot{z}(s)=\lambda(s) f(z(s)), s \in\left(s_{0}-\delta_{1}, s_{0}+\delta_{2}\right)$ that $\lambda(s)$, or rather its extension for complex $s$, is a meromorphic function in a neighborhood of $s_{0}$. On the other hand, since $\lambda(s)=\dot{t}(s), s \in\left(s_{0}, s_{0}+\delta_{1}\right)$ where $t(s)$ is bounded, we have that $\lambda(s)$ cannot have a pole at $s_{0}$ therefore it must be analytic in a neighborhood of $s_{0}$. This implies that by conveniently diminishing the value of $\delta_{1}$ and the value of $\delta_{2}$ if necessary, we have that $\lambda(s), s \in\left(s_{0}-\delta_{1}, s_{0}+\delta_{2}\right)$ is real analytic. By conveniently diminishing the value of $\delta_{1}$ if necessary we can assume without loss of generality that $\lambda(s) \neq 0, s \in\left(s_{0}-\delta_{1}, s_{0}\right)$. Let us assume first that $\lambda>0$. Then the result follows by taking $t=t(s), s \in\left(s_{0}-\delta_{1}, s_{0}+\delta_{2}\right)$ such that $d t / d s=\lambda(s), t\left(s_{0}\right)=t_{0}$ and $t\left(s_{0}-\delta_{1}\right)=t_{2-}$, which defines $t(s), s \in\left(s_{0}-\delta_{1}, s_{0}\right]$ as an as-curve in $\mathbb{R}$ and also $t_{2-}$. Since $\lambda(s)>0, s \in\left(s_{0}-\delta_{1}, s_{0}\right)$ we have that $t(s)$ is an analytic diffeomorphism from $\left(s_{0}-\delta_{1}, s_{0}\right)$ onto $\left(t_{2-}, t_{0}\right)$ which is an as-curve, while $t:\left[s_{0}-\delta_{1}, s_{0}\right] \rightarrow\left[t_{2-}, t_{0}\right]$ is an homeomorphism. Then we can define $x_{2-}(t), t \in\left(t_{2-}, t_{0}\right]$ by $x_{2-}(t)=z(s(t))$ where $s(t)$ is the inverse of $t(s)$. The rest of the proof can be performed in a similar way.

The previous theorem says, in particular, that an as-solution $x_{+}(t), t \in\left[t_{0}, t_{1}\right)$ to a given IDE $(a, f)$ gives rise to an as-solution $x_{-}(t), t \in\left(t_{2-}, t_{0}\right]$ of $(a, f)$ or $(a,-f)$ such that $x_{+}\left(t_{0}\right)=x_{-}\left(t_{0}\right)$ and graph $x_{-} \cup$ graph $x_{+}=\operatorname{graph} z$ for some as-curve $z(s), s \in$ $\left(s_{0}-\delta_{1}, s_{0}+\delta_{2}\right)$, which is a solution to the homogeneous system $a(z) \dot{z}=\dot{t} f(z), s \in$ $\left(s_{0}-\delta_{1}, s_{0}\right) \cup\left(s_{0}, s_{0}+\delta_{2}\right)$, and $x_{-}, x_{+}$are reparametrizations of $z\left|\left(s_{0}-\delta_{1}, s_{0}\right], z\right|\left[s_{0}, s_{0}+\delta_{2}\right)$.

For a given $\operatorname{IDE}(a, f)$ with domain $M$ and range $F$, an as-solution $x(t), t \in\left[t_{0}, t_{1}\right)$ 
$\left(t \in\left(t_{0}, t_{1}\right], t \in\left(t_{0}, t_{1}\right), t \in\left[t_{0}, t_{1}\right]\right)$, to $(a, \pm f)$, in $M$ is, by definition, an as-solution to some of the systems $(a, f)$ or $(a,-f)$, in $M$. The notion of a pas-solution or an lcssolution to $(a, \pm f)$, in $M$ is defined by the condition that each as-piece is an as-solution to $(a, \pm f)$ in $M$.

We have the following extension theorem

Theorem 4.17 Let $(a, f)$ be an IDE with domain $M$ and range $F$. Let $x(t), t \in\left[t_{0}, t_{1}\right]$ be a continuous pas-solution to $(a, f)$, so its graph is compact. Then $x(t)$ can be extended to a continuous pas-solution $\bar{x}(t), t \in\left[\bar{t}_{0}, \bar{t}_{1}\right]$, to $(a, \pm f)$ for some $\bar{t}_{0}<t_{0}<t_{1}<\bar{t}_{1}$.

Proof. If $x$ is a constant the proof is immediate. Let us assume that $x$ is not a constant. We have an as-piece of $x$ of the type $x \mid\left[t_{0}, t_{0}+\delta_{2}\right)$. As a consequence of theorem 4.16] we have that $x \mid\left[t_{0}, t_{0}+\delta_{2}\right)$ can be extended to a continuous pas-solution, say $u(t), t \in\left[t_{2-}, t_{0}+\delta_{2}\right)$, to $(a, \pm f)$, where $u_{+}=x \mid\left[t_{0}, t_{0}+\delta_{2}\right)$ and $u_{-}:\left(\left[t_{2_{-}}, t_{0}\right] \rightarrow M\right.$ are the as-pieces. We can glue the as-piece $u_{-}$to $x$ and take $\bar{t}_{0}=t_{2-}$ to obtain a continuous pas-solution to $(a, \pm f)$ defined in $\left[\bar{t}_{0}, t_{1}\right)$ which extends $x$. We can proceed now in a similar way to obtain, in turn, an extension of this solution to a pas-continuous solution to $(a, \pm f)$ defined in some interval $\left[\bar{t}_{0}, \bar{t}_{1}\right]$.

Corollary 4.18 Let $(a, 0)$ be an homogeneous IDE with domain $M$ and range $F$. Let $x(t), t \in\left[t_{0}, t_{1}\right]$ be a continuous pas-solution to $(a, f)$, so its graph is compact. Then $x(t)$ can be extended to a continuous pas-solution $\bar{x}(t), t \in\left[\bar{t}_{0}, \bar{t}_{1}\right]$, to $(a, 0)$ for some $\bar{t}_{0}<t_{0}<t_{1}<\bar{t}_{1}$.

\section{The example of the symmetric elastic sphere}

As we have said before the main purpose of this paper is not to pursue the investigation of how to obtain systematically desingularizations of given IDE by using systematic procedures to desingularize given closed analytic sets and then apply all this to solve a given IDE by a systematic procedure, but rather to show how having a desingularization of a given IDE may help to understand its solutions. In the next section we study an example from nonholonomic mechanics, namely the symmetric elastic sphere, and show using a desingularization of the equations of motion reduced by the symmetry, which constitute an IDE, how to study the dynamics and solve completely the system by quadratures. 
Preliminaries. Continuing with the description of the symmetric elastic sphere given in section 11 we shall now give a more precise description of this system as a nonholonomic system. For references on nonholonomic mechanics related to rolling spheres see [2, 4, 5, 43, 44, 45] and references therein.

A rigid sphere rolling on a plane can be modelled as a nonholonomic system on the group $S O(3) \times \mathbb{R}^{2}$ where, for a given element $(A, x) \in S O(3) \times \mathbb{R}^{2}, A$ represents a rigid rotation and $x$ the position of the point of contact of the sphere with the plane. Now we will assume that $S O(3) \times \mathbb{R}^{2}$ is also a good configuration space for an elastic sphere where deformations are small and concentrated only near the area of contact which is a small circle whose center has a position given by $x$. The kinematics of this system can be conveniently described as follows. We assume that there is an ortonormal system fixed in the space, say $\left(e_{1}, e_{2}, e_{3}\right), e_{1}=(1,0,0), e_{2}=(0,1,0), e_{3}=(0,0,1)$, then we have a basis moving with the body, $\left(A e_{1}, A e_{2}, A e_{3}\right)$, where $A=A(t)$. We introduce the variable $z \in S^{2}$, given by $z=A e_{3}$. The spatial angular velocity $\omega$ can be written $\omega=v_{0} z+z \times \dot{z}$, so $v_{0}=\langle\omega, z\rangle$ is the component of $\omega$ along $z$. The nonholonomic constraint is given by the nonsliding condition. Since the area of contact of the sphere with the plane is a circle we must add to the usual nonsliding condition $\omega \times r e_{3}=\dot{x}$ for the rigid rolling sphere (see, for instance, [45] where Lagrange-D'Alembert-Poincaré equations for the rigid sphere have been written) where $r$ is the radius of the sphere, the extra condition that the vertical component of the spatial angular velocity is 0 , that is, $\omega_{3}=0$.

We are going to assume that the center of mass coincides with the center of the sphere and that the principal axis of inertia are $\left(A e_{1}, A e_{2}, A e_{3}\right)$. The three principal moments of inertia of the sphere are $I_{1}, I_{2}, I_{3}$, and we are going to assume that $I_{1}=I_{2}$. We introduce the adimensional quantities $\alpha=I_{3} / I_{1}$ and $\beta=M r^{2} / I_{1}$, where $M$ is the mass of the sphere. The Lagrangian of the system is given by the kinetic energy,

$$
\frac{1}{2} I_{1} \dot{z}^{2}+\frac{1}{2} I_{3} v_{0}^{2}+\frac{1}{2} M \dot{x}^{2}
$$

where $\dot{x}$ is the velocity of the center of the sphere. The nonholonomic constraint is given by $\dot{x}=\omega \times r e_{3}$ and $\omega_{3}=0$, and using this we can conclude that the kinetic energy of the actual motion of the symmetric sphere is given by

$$
E=\frac{1}{2}\left(I_{1}+M r^{2}\right) \dot{z}^{2}+\frac{1}{2}\left(I_{3}+M r^{2}\right) v_{0}^{2}
$$

As we have said in the Introduction the addition of the extra condition $\omega_{3}=0$ introduces an extra singularity in the reduced system, which is an IDE, and we need to apply our 
desingularization procedure to obtain a single equivalent differential equation describing the system, in the sense of theorems 4.14 and 4.15. We will show that the desingularizing manifold containing the essential dynamics, in which this differential equation is defined, is diffeomorphic to $S^{2} \times S^{1}$. Another interesting feature of the desingularization method for the example of the symmetric elastic sphere is that integrability by quadratures appears in a natural way, in terms of angular coordinates of $S^{2} \times S^{1}$.

The IDE for the symmetric elastic sphere. As a result of reduction by the symmetry techniques, in this case reduction by the subgroup $S O(2) \times \mathbb{R}^{2}$ we obtain the following system of Lagrange-D'Alembert-Poincaré equations, which is an IDE,

$$
\begin{array}{r}
(\alpha+\beta)\left(z \times e_{3}\right) \dot{v}_{0}+(1+\beta)<z, e_{3}>\nabla_{\dot{z}} \dot{z}- \\
(\alpha+\beta) v_{0}<z, e_{3}>(z \times \dot{z})=0 \\
v_{0}<z, e_{3}>+<z \times \dot{z}, e_{3}>=0 .
\end{array}
$$

Here $\nabla$ represents the Levi-Civita connection on $S^{2}$ with respect to the standard metric. This is a consequence of the methods developed in [5], after some more or less straightforward calculations which we will not explain here. The previous LagrangeD'Alembert-Poincaré equations are derived under the assumption $z_{3} \neq 0$ because the so called dimension assumption adopted in [5] is not satisfied for the whole manifold $S^{2}$. Nevertheless, by continuity, the equations (5.1), (5.2) are also satisfied by the motion of the rolling ball for $z_{3}=0$. Without using the derivation of the Lagrange-D'AlembertPoincaré equations, the careful reader may want to check directly that the previous system of equations, or equivalently, the system of equations (5.8)-(5.15), is equivalent to balance of momentum plus the condition $\omega_{3}=0$.

Since $\nabla_{\dot{z}} \dot{z}=z \times(\ddot{z} \times z)$ by taking the inner product of (15.1) with $\dot{z}$ and using (15.2) we get, at least for $<z, e_{3}>\neq 0$,

$$
0=\frac{d}{d t}\left((1+\beta) \dot{z}^{2}+(\alpha+\beta) v_{0}^{2}\right)
$$

from which one deduces

$$
2 \epsilon=(1+\beta) \dot{z}^{2}+(\alpha+\beta) v_{0}^{2}
$$

where $\epsilon$ represents the normalized energy. This equation represents conservation of energy, as one can check more directly by looking at the expression of the kinetic energy $E$ given at the beginning of this section. We shall assume from now on that $\epsilon>0$, 
otherwise the motion is trivial.

We have the following equations to be satisfied for the symmetric elastic sphere in variables $(z, u)$ where $\dot{z}=v$ and $v \times z=u$, so the variable $v_{0}$ does not appears,

$$
\begin{array}{r}
(1+\beta)<z, e_{3}><\dot{u}, e_{3} \times z>+(\alpha+\beta)<u, e_{3}>^{2}=0 \\
(1+\beta)<z, e_{3}>^{2} u^{2}+(\alpha+\beta)<u, e_{3}>^{2}-2 \epsilon<z, e_{3}>^{2}=0
\end{array}
$$

Equation (5.5) is obtained by taking the inner product of (5.1) with $e_{3}$ and using (5.2) while equation (5.6) is obtained from equation (5.4) and equation (5.2).

Equations (5.5) and (5.6) involve the variables $(z, u) \in T S^{2}$ and we have a natural inclusion $T S^{2} \subseteq S^{2} \times \mathbb{R}^{3}$, where

$$
T S^{2}=\left\{(z, u) \in S^{2} \times \mathbb{R}^{3}:<z, u>=0\right\} .
$$

Equations (5.5), (5.6) form an IDE in the manifold $T S^{2}$. By adding the equation $z^{2}=1$, we obtain an equivalent IDE in the variables $(z, u) \in \mathbb{R}^{3} \times \mathbb{R}^{3}$. Let us include, in addition, the equation of conservation of energy (5.4), written in terms of the variables $\left(z, u, v_{0}\right)$, as follows

$$
2 \epsilon=(1+\beta) u^{2}+(\alpha+\beta) v_{0}^{2}
$$

In other words, we are going to study the system of equations given by (5.5), (5.6), (5.7) for a fixed $\epsilon>0$. Of course equations (5.6) and (5.7) taking into account (5.2) are redundant for $z_{3} \neq 0$, but for $z_{3}=0$ the system given by the equations (5.5), (5.6) includes solutions of the type $z(t)=$ const, with $z_{3}=0$, where $v_{0}$ takes any given value and, since $u=0$ for this kind of motion, the energy is given by $2 \epsilon=(\alpha+\beta)\left(v_{0}\right)^{2}$ and therefore the condition that the energy must have a fixed value will not be satisfied. Of course we can study with our methods both, the system given by (5.5), (5.6), (5.7) and also the system given by (5.5), (5.6), but we will chose to study just the first of them, for simplicity.

The IDE for the symmetric elastic sphere in the standard form. Considering that $z^{2}=1$ and $\dot{z}=z \times u$, we must have $\langle z, u\rangle=0$. Using what was said in the previous paragraph, and according to theorem 2.1 we can write the system of equations 
for the symmetric elastic sphere in variables $\left(z, u, v_{0}\right) \in \mathbb{R}^{3} \times \mathbb{R}^{3} \times \mathbb{R}$ as follows,

$$
\begin{aligned}
\dot{z}_{1} & =z_{2} u_{3}-z_{3} u_{2} \\
\dot{z}_{2} & =z_{3} u_{1}-z_{1} u_{3} \\
\dot{z}_{3} & =z_{1} u_{2}-z_{2} u_{1} \\
0 & =(1+\beta) z_{3}\left(-z_{2} \dot{u}_{1}+z_{1} \dot{u}_{2}\right)+(\alpha+\beta) u_{3}^{2} \\
0 & =(1+\beta) z_{3}^{2}\left(u_{1}^{2}+u_{2}^{2}+u_{3}^{2}\right)+(\alpha+\beta) u_{3}^{2}-2 \epsilon z_{3}^{2} \\
0 & =z_{1}^{2}+z_{2}^{2}+z_{3}^{2}-1 \\
0 & =z_{1} u_{1}+z_{2} u_{2}+z_{3} u_{3} \\
0 & =2 \epsilon-(1+\beta) u^{2}-(\alpha+\beta) v_{0}^{2} .
\end{aligned}
$$

The system (5.8)-(5.15) can be written in the form

$$
a(X) \dot{X}=f(X)
$$

with $X=\left(z, u, v_{0}\right)$, where,

$$
\begin{aligned}
& a\left(z, u, v_{0}\right)=\left[\begin{array}{ccccccc}
1 & 0 & 0 & 0 & 0 & 0 & 0 \\
0 & 1 & 0 & 0 & 0 & 0 & 0 \\
0 & 0 & 1 & 0 & 0 & 0 & 0 \\
0 & 0 & 0 & -(1+\beta) z_{2} z_{3} & (1+\beta) z_{1} z_{3} & 0 & 0 \\
0 & 0 & 0 & 0 & 0 & 0 & 0 \\
0 & 0 & 0 & 0 & 0 & 0 & 0 \\
0 & 0 & 0 & 0 & 0 & 0 & 0 \\
0 & 0 & 0 & 0 & 0 & 0 & 0
\end{array}\right] \\
& f\left(z, u, v_{0}\right)=\left[\begin{array}{c}
z_{2} u_{3}-z_{3} u_{2} \\
z_{3} u_{1}-z_{1} u_{3} \\
z_{1} u_{2}-z_{2} u_{1} \\
-(\alpha+\beta) u_{3}^{2} \\
(1+\beta) z_{3}^{2}\left(u_{1}^{2}+u_{2}^{2}+u_{3}^{2}\right)+(\alpha+\beta) u_{3}^{2}-2 \epsilon z_{3}^{2} \\
z_{1}^{2}+z_{2}^{2}+z_{3}^{2}-1 \\
z_{1} u_{1}+z_{2} u_{2}+z_{3} u_{3} \\
2 \epsilon-(1+\beta) u^{2}-(\alpha+\beta) v_{0}^{2}
\end{array}\right] .
\end{aligned}
$$

Application of the algorithm. We will work on the manifold $M=\mathbb{R}^{7}$, where $\left(z_{1}, z_{2}, z_{3}, u_{1}, u_{2}, u_{3}, v_{0}\right) \in \mathbb{R}^{7}$ are independent variables. Then our IDE is given by 
equations (5.8)-(5.15). We can easily see that $k_{r}=4, S_{4}(M)=M, L_{4}(M)=M_{0}$, $M_{1}=M-L_{4}(M), M_{2}=\emptyset$. Now we shall describe $M_{0}$ by equations. Let

$$
\begin{aligned}
& \varphi_{1}=-(1+\beta) z_{2} z_{3} \\
& \varphi_{2}=(1+\beta) z_{1} z_{3} \\
& \nu_{1}=(1+\beta) z_{3}^{2}\left(u_{1}^{2}+u_{2}^{2}+u_{3}^{2}\right)+(\alpha+\beta) u_{3}^{2}-2 \epsilon z_{3}^{2} \\
& \nu_{2}=z_{1}^{2}+z_{2}^{2}+z_{3}^{2}-1 \\
& \nu_{3}=z_{1} u_{1}+z_{2} u_{2}+z_{3} u_{3} \\
& \nu_{4}=2 \epsilon-(1+\beta) u^{2}-(\alpha+\beta) v_{0}^{2} .
\end{aligned}
$$

As we know $M_{0}=L_{4}(M)$ is given by the condition that $\operatorname{rank}[a, f] \leq 4$. Let

$$
\begin{aligned}
M_{0 a} & =\left\{\varphi_{1}=0, \varphi_{2}=0\right\} \\
& =\left\{z_{3}=0\right\} \cup\left\{z_{1}=0, z_{2}=0\right\} \\
M_{0 b} & =\left\{\nu_{1}=0, \nu_{2}=0, \nu_{3}=0, \nu_{4}=0\right\}, .
\end{aligned}
$$

Then we can easily see that $M_{0}=M_{0 a} \cup M_{0 b}$. The desingularization $M^{1}$ of $M_{0}$ will be the disjoint union of the desingularizations of $M_{0 a}$ and $M_{0 b}$.

The desingularization $M_{a}^{1}$ of $M_{0 a}$ can be described by $M_{a}^{1} \equiv\left\{z_{3}=0\right\} \bigsqcup\left\{z_{1}=0, z_{2}=0\right\}$, where $\bigsqcup$ means disjoint union and the projection $\pi_{0}$ is the identity on each disjoint piece of $M_{a}^{1}$. One can see using (5.8) - (5.15) that the lifted system $\left(a_{1}, f_{1}\right) \mid\left\{z_{3}=0\right\}$ satisfies $z_{3}=0, u_{3}=0, z_{1}^{2}+z_{2}^{2}=1$, which implies $\dot{z}=0$, and also, since $u=\dot{z} \times z$, that $u=0$. This describes the motion completely. It consists of the rolling of the sphere with $z(t)=\left(z_{10}, z_{20}, 0\right)$ fixed and the $z$ component of the angular velocity $v_{0}$ satisfies $2 \epsilon=(\alpha+\beta)\left(v_{0}\right)^{2}$. The lifted system $\left(a_{1}, f_{1}\right) \mid\left\{z_{1}=0, z_{2}=0\right\}$ satisfies $z_{1}=0, z_{2}=0$, $z_{3}= \pm 1$, therefore $\dot{z}=0$, and then $u=0$, which contradicts equation $\nu_{1}=0$, because we have assumed $\epsilon>0$. So there is no motion, that is, no solution, for the system $\left(a_{1}, f_{1}\right) \mid\left\{z_{1}=0, z_{2}=0\right\}$.

Now we will desingularize $M_{0 b}$. We are going to see that $M_{0 b}$ is in fact a nonsingular manifold. More precisely, we will define the desingularizing manifold $M_{b}^{1}$ by equations in the variables $\left(z, u, v_{0}\right)$, with $v_{0} z_{3}=u_{3}$, from (5.2). For simplicity, we call $\mu=2 \epsilon /(1+\beta)>$ 0 and $\lambda=(\alpha+\beta) /(1+\beta)>0$, from now on. Then we have the following equations defining the nonsingular manifold $M_{b}^{1}$, 


$$
\begin{aligned}
& 0=u_{3}-v_{0} z_{3} \\
& 0=u_{1}^{2}+u_{2}^{2}+u_{3}^{2}+\lambda v_{0}^{2}-\mu \\
& 0=z_{1}^{2}+z_{2}^{2}+z_{3}^{2}-1 \\
& 0=z_{1} u_{1}+z_{2} u_{2}+z_{3} u_{3} .
\end{aligned}
$$

See Appendix A for a proof that the system above defines a nonsingular manifold. The map $\pi_{0}: M_{b}^{1} \rightarrow M$ is then given by the restriction of the identity $\left(z, u, v_{0}\right) \rightarrow\left(z, u, v_{0}\right)$ to $M_{b}^{1}$ and one can check that the image of $\pi_{0}$ is precisely $M_{0 b}$.

According to theorem 2.1] the system lifted to $M_{b}^{1}$ has the same solutions as the system given by the equations

$$
\begin{aligned}
\dot{z}_{1} & =z_{2} u_{3}-z_{3} u_{2} \\
\dot{z}_{2} & =z_{3} u_{1}-z_{1} u_{3} \\
\dot{z}_{3} & =z_{1} u_{2}-z_{2} u_{1} \\
z_{2} \dot{u}_{1}-z_{1} \dot{u}_{2} & =\lambda v_{0} u_{3} \\
0 & =u_{3}-v_{0} z_{3} \\
0 & =u_{1}^{2}+u_{2}^{2}+u_{3}^{2}+\lambda v_{0}^{2}-\mu \\
0 & =z_{1}^{2}+z_{2}^{2}+z_{3}^{2}-1 \\
0 & =z_{1} u_{1}+z_{2} u_{2}+z_{3} u_{3} .
\end{aligned}
$$

More precisely, if we define

$$
\tilde{a}\left(z, u, v_{0}\right)=\left[\begin{array}{ccccccc}
1 & 0 & 0 & 0 & 0 & 0 & 0 \\
0 & 1 & 0 & 0 & 0 & 0 & 0 \\
0 & 0 & 1 & 0 & 0 & 0 & 0 \\
0 & 0 & 0 & z_{2} & -z_{1} & 0 & 0 \\
0 & 0 & 0 & 0 & 0 & 0 & 0 \\
0 & 0 & 0 & 0 & 0 & 0 & 0 \\
0 & 0 & 0 & 0 & 0 & 0 & 0 \\
0 & 0 & 0 & 0 & 0 & 0 & 0
\end{array}\right], \tilde{f}\left(z, u, v_{0}\right)=\left[\begin{array}{c}
z_{2} u_{3}-z_{3} u_{2} \\
z_{3} u_{1}-z_{1} u_{3} \\
z_{1} u_{2}-z_{2} u_{1} \\
\lambda v_{0} u_{3} \\
u_{3}-v_{0} z_{3} \\
u_{1}^{2}+u_{2}^{2}+u_{3}^{2}+\lambda v_{0}^{2}-\mu \\
z_{1}^{2}+z_{2}^{2}+z_{3}^{2}-1 \\
z_{1} u_{1}+z_{2} u_{2}+z_{3} u_{3}
\end{array}\right]
$$

we see that the system (5.29)-(5.36) is in the form $\tilde{a}(y) \dot{y}=\tilde{f}(y)$, with $y=\left(z, u, v_{0}\right)$, so it is an IDE in standard form with domain $\mathbb{R}^{7}$ and range $\mathbb{R}^{8}$, and our IDE in $M_{b}^{1}$ is given 
by the restriction $\left(a_{1}, f_{1}\right)=(\tilde{a}, \tilde{f}) \mid M_{b}^{1}$. In order to continue with the algorithm, we shall find explicitly the lifted system $\left(a_{1}, f_{1}\right)$. By differentiating the equations (5.25)-(5.28), eliminating the redundant equation $z_{1} \dot{z}_{1}+z_{2} \dot{z}_{2}+z_{3} \dot{z}_{3}=0$, also realizing appropriate linear operations in the range space, that is, using projections as explained in section 2 . and also according to theorem [2.1, we have the following system with domain $\mathbb{R}^{7}$ and range $\mathbb{R}^{11}$, which is also equivalent to our system $\left(a_{1}, f_{1}\right)$,

$$
\begin{aligned}
\dot{z}_{1} & =z_{2} u_{3}-z_{3} u_{2} \\
\dot{z}_{2} & =z_{3} u_{1}-z_{1} u_{3} \\
\dot{z}_{3} & =z_{1} u_{2}-z_{2} u_{1} \\
z_{2} \dot{u}_{1}-z_{1} \dot{u}_{2} & =\lambda v_{0} u_{3} \\
z_{1} \dot{u}_{1}+z_{2} \dot{u}_{2}+z_{3} \dot{u}_{3} & =0 \\
u_{1} \dot{u}_{1}+u_{2} \dot{u}_{2}+u_{3} \dot{u}_{3}+\lambda v_{0} \dot{v}_{0} & =0 \\
\dot{u}_{3}-z_{3} \dot{v}_{0} & =v_{0} z_{1} u_{2}-v_{0} z_{2} u_{1} \\
0 & =u_{3}-v_{0} z_{3} \\
0 & =u_{1}^{2}+u_{2}^{2}+u_{3}^{2}+\lambda v_{0}^{2}-\mu \\
0 & =z_{1}^{2}+z_{2}^{2}+z_{3}^{2}-1 \\
0 & =z_{1} u_{1}+z_{2} u_{2}+z_{3} u_{3} .
\end{aligned}
$$

This system is still not completely desingularized. One can check by direct calculation that it can be desingularized in two more iterations of the algorithm. However, in this example there is an interesting alternative to find the solutions, which starts with a precise description of the manifold $M_{b}^{1}$. We prefer this alternative because having an identification of $M_{b}^{1}$ also helps to understand the dynamics in a direct way, as we will see soon. This is precisely one of the points of the present work, namely, to show how in examples the desingularization method may helps to understand the dynamics of a given system.

Identification of $M_{b}^{1}$. The manifold $M_{b}^{1}$ is given by the equations (5.25) - (15.28) in the space of the variables $\left(z_{1}, z_{2}, z_{3}, u_{1}, u_{2}, u_{3}, v_{0}\right)$, as we have seen before. The equation (5.28) tells us that $u$ is a vector tangent to the 2 -sphere $S^{2}$, given by $z^{2}-1=0$. Heuristically, for each $z \in S^{2}$ we consider the 3 dimensional space $T_{z} S^{2} \times R_{z}$, where $R_{z}$ represents a line normal to the sphere at $z \in S^{2}$, so the collection of all $R_{z}$ is a trivial real line vector bundle with base $S^{2}$. Equation (5.25) is a plane containing the origin $0=0_{z}$ since $z_{3}$ is fixed once $z$ is fixed. Equation (5.26) gives an ellipsoid. The intersection of the plane with the ellipsoid is an ellipse. Therefore $M_{b}^{1}$ must be some fiber bundle with fiber $S^{1}$ and base $S^{2}$. Using all this and some imagination we can see that it is, 
in fact, the trivial bundle $S^{2} \times S^{1}$, moreover, we have the following parametrization of $M_{b}^{1}$ in variables $(\theta, \varphi, \psi)$. In any case, this assertion can be easily checked after some straightforward calculations.

$$
\begin{aligned}
& z_{1}=\sin \theta \cos \varphi \\
& z_{2}=\sin \theta \sin \varphi \\
& z_{3}=\cos \theta \\
& u_{1}=-a \cos (\varphi-\psi) \cos ^{2} \theta \cos \varphi-b \sin (\varphi-\psi) \sin \varphi \\
& u_{2}=-a \cos (\varphi-\psi) \cos ^{2} \theta \sin \varphi+b \sin (\varphi-\psi) \cos \varphi \\
& u_{3}=a \cos (\varphi-\psi) \cos \theta \sin \theta \\
& v_{0}=a \cos (\varphi-\psi) \sin \theta,
\end{aligned}
$$

where

$$
a=\sqrt{\frac{\mu}{\lambda \sin ^{2} \theta+\cos ^{2} \theta}}, \quad b=\sqrt{\mu}^{\prime} .
$$

In other words, by some straightforward calculations we can check that $\left(z_{1}, z_{2}, z_{3}, u_{1}, u_{2}, u_{3}, v_{0}\right)$ in coordinates $(\theta, \varphi, \psi)$ satisfies (5.25) $-(5.28)$.

We can see that equations (5.48)-(15.54) define a diffeomorphism $f: S^{2} \times S^{1} \rightarrow M_{b}^{1}$, $f(z,(\cos \psi, \sin \psi))=\left(z, u, v_{0}\right)$, which gives the desired identification of $M_{b}^{1}$. See Appendix $\mathbf{B}$ for a proof that $f$ is a diffeomorphism.

The differential equation for the symmetric elastic sphere in $M_{b}^{1}$ in variables $(\theta, \varphi, \psi)$. Considering the parametrization for $M_{b}^{1}$ given by (5.48)-(5.54) we get the equations for (5.29)-(5.36) in coordinates $(\theta, \varphi, \psi)$,

$$
\begin{aligned}
\cos \theta \cos \varphi \dot{\theta}-\sin \theta \sin \varphi \dot{\varphi}= & a \cos \theta \sin \varphi \cos (\varphi-\psi)- \\
& -b \cos \theta \cos \varphi \sin (\varphi-\psi) \\
\cos \theta \sin \varphi \dot{\theta}+\sin \theta \cos \varphi \dot{\varphi}= & -a \cos \theta \cos \varphi \cos (\varphi-\psi)- \\
-\sin \theta \dot{\theta}= & -b \sin \theta \sin (\varphi-\psi) \\
a \sin \theta \cos ^{2} \theta \cos (\varphi-\psi) \dot{\varphi}-= & \\
-b \sin \theta \cos (\varphi-\psi)(\dot{\varphi}-\dot{\psi})= & \lambda a^{2} \cos ^{2}(\varphi-\psi) \sin ^{2} \theta \cos \theta
\end{aligned}
$$


If $\sin \theta \neq 0$ the system (5.55) $-(15.58)$ becomes

$$
\begin{aligned}
\dot{\theta} & =-b \sin (\varphi-\psi) \\
\dot{\varphi} & =-a \frac{\cos \theta}{\sin \theta} \cos (\varphi-\psi) \\
\dot{\psi} & =a \cos (\varphi-\psi) \frac{\cos \theta}{\sin \theta}\left(\frac{b}{a}-1\right),
\end{aligned}
$$

or equivalently,

$$
\begin{aligned}
\dot{\theta} & =-b \sin (\varphi-\psi) \\
\dot{\varphi} & =-a \frac{\cos \theta}{\sin \theta} \cos (\varphi-\psi) \\
\dot{\psi} & =(b-a) \frac{\cos \theta}{\sin \theta} \cos (\varphi-\psi)
\end{aligned}
$$

It can be easily seen that this system can be integrated by quadratures. For instance, if we call $w=\varphi-\psi$, we can write (5.62)-(5.64) as a planar system in coordinates $(\theta, w)$,

$$
\begin{aligned}
\dot{\theta} & =-b \sin w \\
\dot{w} & =-b \frac{\cos \theta}{\sin \theta} \cos w,
\end{aligned}
$$

which in turn leads to the separable equation

$$
\frac{d \theta}{d w}=\tan \theta \tan w
$$

Of course the system (5.55) - (5.58) is still an analytic IDE on the analytic manifold $S^{2} \times S^{1}$ which is of constant rank for $\sin \theta \neq 0$, and the rank changes for $\sin \theta=0$. So we should continue the desingularization process, which is not difficult but we are not going to explain the details. Instead, we simply observe that it is not difficult to see, alternatively, that the only solution with some initial condition compatible with the system and involving the condition $\sin \theta=0$, that is, initial condition of the type $\left(z_{10}, z_{20}, z_{30}, u_{10}, u_{20}, u_{30}, v_{00}\right)=\left(0,0, \pm 1, u_{10}, u_{20}, 0,0\right)$, consists of a uniform circular motion of $z$ on a vertical plane perpendicular to the constant vector $\left(u_{1}(t), u_{2}(t), u_{3}(t)\right)=$ $\left(u_{10}, u_{20}, 0\right)$, while $v_{0}(t)=0$. This is also consistent with physical reasoning.

We are not going to perform a detailed study of the dynamics of the symmetric elastic sphere in this paper, although it is clear that having the kind of explicit solutions that we have found clearly helps to study the standard dynamical systems questions about equilibria, linear and nonlinear stability, bifurcations, and, also control of the system, with potential applications to robotics. A future work in this direction is being planed. 
Appendix A. We will prove that $M_{b}^{1}$ is a nonsingular 3-dimensional manifold. We must prove that the matrix

$$
\left[\begin{array}{ccccccc}
0 & 0 & -v_{0} & 0 & 0 & 1 & -z_{3} \\
0 & 0 & 0 & u_{1} & u_{2} & u_{3} & \lambda v_{0} \\
z_{1} & z_{2} & z_{3} & 0 & 0 & 0 & 0 \\
u_{1} & u_{2} & u_{3} & z_{1} & z_{2} & z_{3} & 0
\end{array}\right]
$$

has rank 4 , for all $\left(z, u, v_{0}\right) \in M_{b}^{1}$.

1. If $z_{3}= \pm 1, z_{1}=z_{2}=u_{3}=v_{0}=0,\left(u_{1}, u_{2}\right) \neq 0$, we get

$$
\left[\begin{array}{ccccccc}
0 & 0 & 0 & 0 & 0 & 1 & \pm 1 \\
0 & 0 & 0 & u_{1} & u_{2} & 0 & 0 \\
0 & 0 & \mp 1 & 0 & 0 & 0 & 0 \\
u_{1} & u_{2} & 0 & 0 & 0 & \mp 1 & 0
\end{array}\right]
$$

which has rank 4.

2. If $z_{3}=0, u_{3}=0,\left(z_{1}, z_{2}\right) \neq 0, z_{1} u_{1}+z_{2} u_{2}=0$, we have

$$
\left[\begin{array}{ccccccc}
0 & 0 & -v_{0} & 0 & 0 & 1 & 0 \\
0 & 0 & 0 & u_{1} & u_{2} & 0 & \lambda v_{0} \\
z_{1} & z_{2} & 0 & 0 & 0 & 0 & 0 \\
u_{1} & u_{2} & 0 & z_{1} & z_{2} & 0 & 0
\end{array}\right]
$$

(a) If $v_{0}=0$, then $\left(u_{1}, u_{2}\right) \neq 0$. For instance, if $u_{1} \neq 0$ we have the subdeterminant

$$
\left|\begin{array}{cccc}
0 & 0 & 0 & 1 \\
0 & 0 & u_{1} & 0 \\
z_{1} & z_{2} & 0 & 0 \\
u_{1} & u_{2} & z_{1} & 0
\end{array}\right|=-u_{1}\left|\begin{array}{cc}
z_{1} & z_{2} \\
u_{1} & u_{2}
\end{array}\right| \neq 0
$$

In fact, if

$$
\left|\begin{array}{ll}
z_{1} & z_{2} \\
u_{1} & u_{2}
\end{array}\right|=0
$$

then

$$
\begin{array}{r}
-z_{2} u_{1}+z_{1} u_{2}=0 \\
z_{1} u_{1}+z_{2} u_{2}=0
\end{array}
$$


and since $z_{1}^{2}+z_{2}^{2}=1$, we have $\left(u_{1}, u_{2}\right)=0$, a contradiction. A similar result holds if $u_{2} \neq 0$.

(b) If $v_{0} \neq 0$, and, say $z_{1} \neq 0$, we have

$$
\left|\begin{array}{cccc}
0 & -v_{0} & 0 & 0 \\
0 & 0 & u_{1} & \lambda v_{0} \\
z_{1} & 0 & 0 & 0 \\
u_{1} & 0 & z_{1} & 0
\end{array}\right|=\lambda v_{0}\left|\begin{array}{ccc}
0 & -v_{0} & 0 \\
z_{1} & 0 & 0 \\
u_{1} & 0 & z_{1}
\end{array}\right|=-\lambda v_{0}^{2} z_{1}^{2} \neq 0
$$

A similar result holds if $z_{2} \neq 0$.

3. Assume now that $0<\left|z_{3}\right|<1 ;\left(z_{1}, z_{2}\right) \neq 0$.

(a) If $v_{0}=0 ; u_{3}=0,\left(u_{1}, u_{2}\right) \neq 0, z_{1} u_{1}+z_{2} u_{2}=0$, we have

$$
\left[\begin{array}{ccccccc}
0 & 0 & 0 & 0 & 0 & 1 & -z_{3} \\
0 & 0 & 0 & u_{1} & u_{2} & 0 & 0 \\
z_{1} & z_{2} & z_{3} & 0 & 0 & 0 & 0 \\
u_{1} & u_{2} & 0 & z_{1} & z_{2} & z_{3} & 0
\end{array}\right]
$$

If, for instance, $u_{1} \neq 0$, we have

$$
\left|\begin{array}{cccc}
0 & 0 & 0 & -z_{3} \\
0 & 0 & u_{1} & 0 \\
z_{1} & z_{2} & 0 & 0 \\
u_{1} & u_{2} & z_{1} & 0
\end{array}\right|=z_{3} u_{1}\left|\begin{array}{cc}
z_{1} & z_{2} \\
u_{1} & u_{2}
\end{array}\right| \neq 0
$$

In fact, if

$$
\left|\begin{array}{ll}
z_{1} & z_{2} \\
u_{1} & u_{2}
\end{array}\right|=0
$$

then $-z_{2} u_{1}+z_{1} u_{2}=0$ and this together with $z_{1} u_{1}+z_{2} u_{2}=0$ gives $\left(u_{1}, u_{2}\right)=0$ or $\left(z_{1}, z_{2}\right)=0$, a contradiction. Similarly if $u_{2} \neq 0$. 
(b) If $v_{0} \neq 0 ; u_{3} \neq 0, z_{1} u_{1}+z_{2} u_{2} \neq 0,\left(u_{1}, u_{2}\right) \neq 0$; in this case the matrix (5.68) also has rank 4 . In fact, $\left(z_{1}, z_{2}, z_{3}\right)$ and $\left(u_{1}, u_{2}, u_{3}\right)$ must be linearly independent, otherwise the condition $z_{1} u_{1}+z_{2} u_{2}+z_{3} u_{3}=0$ would imply $u=0$. Then the last two rows of (5.68) are linearly independent. Using this we can see easily that the second row cannot be a linear combination of the last two rows, so the last three rows are linearly independent. Let $R_{i}$, $i=1,2,3,4$ represent the four rows. If the rank of the matrix is not 4 then we must have $R_{1}=\chi R_{4}+\psi R_{3}+\varphi R_{2}$. This leads to the systems of equations

$$
\begin{aligned}
& \chi u_{1}+\psi z_{1}=0 \\
& \chi u_{2}+\psi z_{2}=0 \\
& \chi u_{3}+\psi z_{3}=-v_{0}
\end{aligned}
$$

and

$$
\begin{aligned}
& \chi z_{1}+\varphi u_{1}=0 \\
& \chi z_{2}+\varphi u_{2}=0 \\
& \chi z_{3}+\varphi u_{3}=1 .
\end{aligned}
$$

Using $\langle z, u\rangle=0$ we can deduce from the first system that $\chi u^{2}=-v_{0} u_{3}$ and since $u_{3}=v_{0} z_{3}$ we obtain $\chi u^{2}=-v_{0}^{2} z_{3}$. On the other hand, using $\langle z, u\rangle=0$ we can deduce from the second system that $\chi=z_{3}$. We can conclude that $u^{2}=-v_{0}^{2}$, which implies $u=v_{0}=0$, a contradiction.

Therefore, also in this case the rank is 4 .

We can conclude that $M_{b}^{1}$ is a nonsingular manifold of dimension 3, defined regularly in $\mathbb{R}^{7}$ by the equations (5.25)-(5.28).

Appendix B. The proof that $f$ is a diffeomorphism goes as follows.

\section{Injectivity}

(a) $z_{3} \neq \pm 1$.

For given $(z,(\cos \psi, \sin \psi)) \in S^{2} \times S^{1}$, we have $\left(z, u, v_{0}\right)=f(z,(\cos \psi, \sin \psi))$. We have that $(\theta, \varphi) \in(0, \pi) \times[0,2 \pi)$ is uniquely determined, moreover, $0<$ $\sin \theta$ and $-1<\cos \theta<1$ are determined. Therefore from equation (5.54) $\cos (\varphi-\psi)$ is determined. Using (5.51) and (5.52) we obtain $\sin (\varphi-\psi)$. Since $z$ determines $(\cos \varphi, \sin \varphi)$, we get $\psi \in[0,2 \pi)$ uniquely determined. 
(b) $z_{3}= \pm 1$.

In this case $\sin \theta=0, \cos \theta= \pm 1, a=b=\sqrt{\mu}$. Using (5.51) and (5.52) we obtain

$$
\begin{aligned}
& u_{1}=-\sqrt{\mu} \cos \psi \\
& u_{2}=-\sqrt{\mu} \sin \psi
\end{aligned}
$$

which determines $\psi \in[0,2 \pi)$.

2. We must prove now that the rank of the tangent map $T_{(z,(\cos \psi, \sin \psi))} f$ is 3 for all $(z,(\cos \psi, \sin \psi)) \in S^{2} \times S^{1}$. We have that the Jacobian matrix calculated at $(\theta, \varphi, \psi)$ of the map given by (5.48)-(5.54) is

$J(\theta, \varphi, \psi)=\left[\begin{array}{ccc}\cos \theta \cos \varphi & -\sin \theta \sin \varphi & 0 \\ \cos \theta \sin \varphi & \sin \theta \cos \varphi & 0 \\ -\sin \theta & 0 & 0 \\ J_{41} & J_{42} & J_{43} \\ J_{51} & J_{52} & J_{53} \\ J_{61} & J_{62} & J_{63} \\ a^{\prime} \cos (\varphi-\psi) \sin \theta+a \cos (\varphi-\psi) \cos \theta & -a \sin (\varphi-\psi) \sin \theta & a \sin (\varphi-\psi) \sin \theta\end{array}\right]$

where we have written

$$
\begin{aligned}
J_{41}= & -a^{\prime} \cos (\varphi-\psi) \cos ^{2} \theta \cos \varphi+2 a \cos (\varphi-\psi) \cos \theta \sin \theta \cos \varphi \\
J_{42}= & a \sin (\varphi-\psi) \cos ^{2} \theta \cos \varphi+a \cos (\varphi-\psi) \cos ^{2} \theta \sin \varphi- \\
& -b \cos (\varphi-\psi) \sin \varphi-b \sin (\varphi-\psi) \cos \varphi \\
J_{43}= & -a \sin (\varphi-\psi) \cos ^{2} \theta \cos \varphi+b \cos (\varphi-\psi) \sin \varphi \\
J_{51}= & -a^{\prime} \cos (\varphi-\psi) \cos ^{2} \theta \sin \varphi+2 a \cos (\varphi-\psi) \cos \theta \sin \theta \sin \varphi \\
J_{52}= & a \sin (\varphi-\psi) \cos ^{2} \theta \sin \varphi-a \cos (\varphi-\psi) \cos ^{2} \theta \cos \varphi+ \\
& +b \cos (\varphi-\psi) \cos \varphi-b \sin (\varphi-\psi) \sin \varphi \\
J_{53}= & -a \sin (\varphi-\psi) \cos { }^{2} \theta \sin \varphi- \\
& -b \cos (\varphi-\psi) \cos \varphi \\
J_{61}= & a^{\prime} \cos (\varphi-\psi) \cos \theta \sin \theta+a \cos (\varphi-\psi)\left[-\sin ^{2} \theta+\cos ^{2} \theta\right] \\
J_{62}= & -a \sin (\varphi-\psi \cos \theta \sin \theta \\
J_{63}= & a \sin (\varphi-\psi) \cos \theta \sin \theta
\end{aligned}
$$

with $a^{\prime}=d a / d \theta$.

(a) If $z_{3}= \pm 1$ then $\theta=0$ or $\theta=\pi$. Let us consider the case $\theta=0$ only since the case $\theta=\pi$ can be treated in a similar way. We cannot use the polar coordinates representations, in fact, we can see that the rank of $J(0, \varphi, \psi)$ and 
$J(\pi, \varphi, \psi)$ is less than 3 for all $(\varphi, \psi)$. On the other hand, we can easily check that for fixed $\psi, f(\theta(t), \varphi, \psi)$ represent the same point in the manifold $M_{b}^{1}$ for $\theta(t)=0$ and $\varphi$ arbitrary, which is the point $(0,0,1,-b \cos \psi,-b \sin \psi, 0,0)$. Let, for instance, $\theta(t)=t$, then the tangent vector to the curve $f(\theta(t), \varphi, \psi)$ at $t=0$, is $(\cos \varphi, \sin \varphi, 0,0,0, b \cos (\varphi-\psi), b \cos (\varphi-\psi))$. For $\varphi=0$ and $\varphi=$ $\pi / 2$ we obtain the linearly independent vectors. $A=(1,0,0,0,0, b \cos \psi, b \cos \psi)$ and $B=(0,1,0,0,0, b \sin \psi, b \sin \psi)$, respectively. On the other hand, the tangent vector to the curve $f(0,0, \psi+t)$ at $t=0$, which is the last column of $J(0,0, \psi)$, is $C=(0,0,0, b \sin \psi,-b \cos \psi, 0,0)$. The three vectors $A, B, C$ are linearly independent for all $\psi$, which shows that the rank of $T_{\left(e_{3},(\cos \psi, \sin \psi)\right)} f$ is 3 for all $\psi$.

(b) If $z_{3}=0, \cos \theta=0$, then $\theta=\pi / 2$ and $a=\sqrt{\mu / \lambda}$. We get in this case

$$
J(\pi / 2, \varphi, \psi)=\left[\begin{array}{ccc}
0 & -\sin \varphi & 0 \\
0 & \cos \varphi & 0 \\
-1 & 0 & 0 \\
0 & -b \sin (2 \varphi-\psi) & b \cos (\varphi-\psi) \sin \varphi \\
0 & b \cos (2 \varphi-\psi) & -b \cos (\varphi-\psi) \cos \varphi \\
-a \cos (\varphi-\psi) & 0 & 0 \\
0 & -a \sin (\varphi-\psi) & a \sin (\varphi-\psi)
\end{array}\right]
$$

i. If $\sin \varphi=0$, then $\cos \varphi= \pm 1$.

Case I: $\sin (\varphi-\psi) \neq 0$, then the rank of $J$ is 3 .

Case II: $\sin (\varphi-\psi)=0$, then $\cos (\varphi-\psi)= \pm 1$ and $-b \cos (\varphi-\psi) \cos \varphi \neq 0$; then the rank of $J$ is 3 .

ii. If $\sin \varphi= \pm 1$, then $\cos \varphi=0$.

Case I: $\sin (\varphi-\psi) \neq 0$, then the rank of $J$ is 3 .

Case II: $\sin (\varphi-\psi)=0$, then $\cos (\varphi-\psi)= \pm 1$ and $-b \cos (\varphi-\psi) \sin \varphi \neq 0$; then the rank of $J$ is 3 .

iii. If $0<|\sin \varphi|<1$,

Case I: $\sin (\varphi-\psi) \neq 0$, then the rank of $J$ is 3 .

Case II: $\sin (\varphi-\psi)=0$, then $\cos (\varphi-\psi)= \pm 1$ and $b \cos (\varphi-\psi) \sin \varphi \neq 0$; then the rank of $J$ is 3 .

(c) If $0<\left|z_{3}\right|<1$, then we have

$$
\left|\begin{array}{ll}
J_{11} & J_{12} \\
J_{21} & J_{22}
\end{array}\right|=\left|\begin{array}{cc}
\cos \theta \cos \varphi & -\sin \theta \sin \varphi \\
\cos \theta \sin \varphi & \sin \theta \cos \varphi
\end{array}\right|=\cos \theta \sin \theta \neq 0 ;
$$

then the rank of $\mathrm{J}$ will be 3 if $J_{i 3} \neq 0$, for at least one $i, 4 \leq i \leq 7$. 
i. If $J_{73} \neq 0$, then the rank of $J$ is 3 .

ii. If $J_{73}=0$, then $\sin (\varphi-\psi)=0$ and $\cos (\varphi-\psi)= \pm 1$. Moreover, $J_{43}=$ $\pm b \sin \varphi$ and $J_{53}= \pm a \cos ^{2} \theta \sin \varphi \pm b \cos \varphi$.

Case I: If $\sin \varphi \neq 0$, then the rank of $J$ is 3 .

Case II: If $\sin \varphi=0, \cos \varphi= \pm 1$ and $J_{53}= \pm b \neq 0$, then the rank of $J$ is 3.

Acknowledgements We thank Jerrold Marsden and Tudor Ratiu for helpful comments and references. The first author also thanks the Bernoulli Center, at EPFL, for its kind hospitality during the last period of the realization of this work and also the Universidad Carlos III de Madrid for its kind hospitality during part of the realization of this work.

\section{References}

[1] Abraham, R. and J.E. Marsden, Foundations of Mechanics, Benjamin, 1978.

[2] Marsden, J.E. and T.S. Ratiu, Introduction to Mechanics and Symmetry, Springer-Verlag, 1994.

[3] H. Cendra, J.E. Marsden, T.S. Ratiu, Lagrangian Reduction by Stages, Memoirs of the AMS 152 (722) (2001).

[4] A.M. Bloch, P.S. Krishnaprasad, J.E. Marsden, R.M. Murray, Nonholonomic Mechanical Systems with Symmetry, Arc. Rat. Mech. Anal. 136 (1996) 21-99.

[5] H. Cendra, J.E. Marsden, T.S. Ratiu, Geometric Mechanics, Lagrangian Reduction and Nonholonomic Systems, in: Mathematics Unlimited-2001 and Beyond, Springer, pp. 221-273.

[6] J. Cortes Monforte, Geometric, Control and Numerical Aspects of Nonholonomic Systems, Sringer, 2002.

[7] P.A.M. Dirac, Generalized Hamiltonian Dynamics, Can. J. of Math. 2 (1950) 129-148.

[8] R. Newcomb, The semistate description of nonlinear and time-variable circuits, IEEE Trans. Circ. Syst. 28 (1981) 62-71. 
[9] V. Venkatasubramanian, H. Schattler, J. Zaborsky, Local bifurcations and feasibility regions in differential-algebraic systems, IEEE Trans. Autom. Control 40 (12) (1995) 1992-2013.

[10] N. McClamroch, H. Krishnan, Nonstandard singularly perturbed control systems and differential-algebraic equations, Int. J. Cont. 55 (4) (1992) 12391253 .

[11] A. Kumar and P. Daoutidis, A DAE Framework for Modelling and Control of Reactive Columns, 4th IFAC Symp. on Dyn. Cont. Chem. Reactors, Helsingor, Denmark (1995) 99-104.

[12] H. Krischnan and H. McClamroch, Tracking in Nonlinear DifferentialAlgebraic Control Systems with Applications to Constrained Robot Systems, Automatica 30 (1994) 1885-1897.

[13] P. Rabier and W. Rheinboldt, Treatment of Implicit differential-algebraic Equations, J. Differential Equations 109 (1994) 110-146.

[14] W. Rheinboldt, Differential algebraic systems as differential equations on manifolds, Math. of Comp. 43 (1984) 473-482.

[15] S. Reich, On a geometrical interpretation of differential-algebraic equations, Circ. Syst. Sign. Proc. 9 (1990) 367-382.

[16] S. Reich, On an existence and uniqueness theory for nonlinear differential algebraic equations, Circ. Syst. Sign. Proc. 10 (1991) 343-359.

[17] P. Rabier and W. Rheinboldt, A general existence and uniqueness theory for implicit differential algebraic equations, Diff. Int. Equations. 4 (1991) 563-582.

[18] A. Szatkowski, Geometric Characterization of Singular Differential Algebraic Equations, Int. J. Syst. Sci. 23 (1992) 167-186.

[19] F. Leon Pritchard, On implicit systems of differential equations, J. Differential Equations 194 (2003) 328-263.

[20] G. Reibig, On Singularities of Autonomous Implicit Ordinary Differential Equations, IEEE Trans. Circ. Syst. 50 (2003) 922-931.

[21] J. Sotomayor, Impasse Singularities of Differential Systems of the Form $A(x) x^{\prime}=F(x)$, J. Differential Equations 169 (2001) 567-587. 
[22] A. Guzman Gomez, Constrained Equations with Impasse Points, J. Math. Anal. Appl. 214 (1997) 292-306.

[23] P. Rabier, W. Rheinboldt, On the computation of impasse points of quasilinear differential-algebraic equations, Math. Comp. 62 (1994) 133-154.

[24] H. Hironaka, Resolution of an algebraic variety over a field of characteristic zero, Ann. of Math. 79 (1964) 109-203.

[25] H. Hauser, The Hironaka Theorem on Resolution of Singularities, Bulletin of the AMS 40 (2003) 323-403.

[26] S. Encinas and O. Villamayor, A new proof of desingularization over fields of characteristic zero, Rev. Mat. Iber. 19 (2003) 339-353.

[27] E. Bierstone, P. D. Milman, Canonical desingularization in characteristic zero by blowing up the maximum strata of a local invariant, Invent. Math 128 (1997) 207-302.

[28] E. Bierstone, P. D. Milman, Desingularization algorithms I. Role of exceptional divisors. Moscow Math. Journal, Volume 3 (3) (2003) 751-805.

[29] H. Hironaka, Subanalytic Sets, Number Theory, Algebraic Geometry and Commutative Algebra, Tokio, Kinokuniya (1973) 453-493.

[30] E. Bierstone and P.D. Milman, Semianalytic and subanalytic sets, Publications mathemátiques de l'I.H.E.S., tome 67 (1988) 5-42.

[31] H.J. Sussmann, Some optimal control applications of real-analytic stratifications and desingularization, Singularities Symposium-Lojasiewicz70, Banach Center Publ. 44 (1998), Polish. Acad. Sci., Warsaw.

[32] H.J. Sussmann, Real analytic desingularization and subanalytic sets: an elementary approach, Trans. Amer. Math. Soc. 317 (2) (1990) 417-461.

[33] L.E. Veselova, New cases of the integrability of the equations of a rigid body in the presence of a nonholonomic constraint, Geometry, differential equations and mechanics, Moscow (1985) 64-68.

[34] Abraham, R., J.E. Marsden and T.S. Ratiu, Manifolds, Tensor Analysis and Applications, Springer-Verlag, 1988. 
[35] P. Rabier, W. Rheinboldt, Classical and generalized solutions of timedependent linear DAEs., Lin. Alg. Appl. 245 (1996) 259-293.

[36] S. Lojasiewicz, Sur le Probléme de la division, Studia Math.18 (1959) 87-136.

[37] S. Lojasiewicz, Triangulation of Semianalytic sets, Ann. Scuola. Norm. Sup. Pisa 18, (1964) 449-474.

[38] S. Lojasiewicz, Sur les ensembles semi-analytiques, Actes du Congress International des Mathematiciens, Nice (1970), Tome 2, 237-241.

[39] A. M. Gabrielov, Formal relations between analytic functions, Math. USSR Izvestija 7 (1973) 1056-1088.

[40] R.M. Hardt, Stratifications of real analytic mappings and images, Inven. Mathem. 28 (1975) 193-208.

[41] R.M. Hardt, Triangulation of subanalytic sets and proper light subanalytic maps, Inven. Mathem. 38 (1977) 207-217.

[42] H. Sussmann, Orbits of families of vector fields and integrability of distributions, Trans. Amer. Math. Soc. 180 (1973) 171-188.

[43] Neimark, Ju. I. and N. A. Fufaev, Dynamics of Nonholonomic Systems, Translations of Mathematical Monographs 33 AMS, Providence RI (1972).

[44] H. Cendra, A. Ibort, M. de León, D. Martin de Diego, A generalized Chetaev's principle for a class of higher order nonholonomic constraints, J. Math. Phys. 45 (7) (2004) 2785-2801.

[45] H. Cendra, E.A. Lacomba, W.A. Reartes, The Lagrange D'AlembertPoincaré equations for the symmetric rolling sphere, Actas del VI Congreso Antonio, A.R Monteiro, Universidad Nacional del Sur, Bahía Blanca (2002) 19-32. 